J. Phys. IV France 130 (2005) 87-113

(C) EDP Sciences, Les Ulis

DOI: $10.1051 /$ jp4:2005130007

\title{
Vibrations et relaxations dans les molécules biologiques. Apports de la diffusion incohérente inélastique de neutrons
}

\author{
J.-M. Zanotti ${ }^{1}$ \\ ${ }^{1}$ Laboratoire Léon Brillouin (CEA-CNRS), CEA Saclay, \\ 91191 Gif-sur-Yvette Cedex, France
}

\begin{abstract}
Résumé. Le présent document ne se veut pas un article de revue mais plutôt un élément d'initiation à une technique encore marginale en Biologie. Le lecteur est supposé être un non spécialiste de la diffusion de neutrons poursuivant une thématique à connotation biologique ou biophysique mettant en jeu des phénomènes dynamiques.

En raison de la forte section de diffusion incohérente de l'atome d'hydrogène et de l'abondance de cet élément dans les protéines, la diffusion incohérente inélastique de neutrons est une technique irremplaçable pour sonder la dynamique interne des macromolécules biologiques. Après un rappel succinct des éléments théoriques de base, nous décrivons le fonctionnement de différents types de spectromètres inélastiques par temps de vol sur source continue ou pulsée et discutons leurs mérites respectifs.

Les deux alternatives utilisées pour décrire la dynamique des protéines sont abordées: (i)l'une en termes de physique statistique, issue de la physique des verres, (ii) la seconde est une interprétation mécanistique. Nous montrons dans ce cas, comment mettre à profit les complémentarités de domaines en vecteur de diffusion et de résolution en énergie de différents spectromètres inélastiques de neutrons (temps de vol, backscattering et spin-écho) pour accéder, à l'aide d'un modèle physique simple, à la dynamique des protéines sur une échelle de temps allant d'une fraction de picoseconde à quelques nanosecondes.
\end{abstract}

Table des matières

1 Introduction

2 La diffusion incohérente inélastique de neutrons aspects théoriques et techniques

2.1 Rappels théoriques $\ldots \ldots \ldots \ldots \ldots$

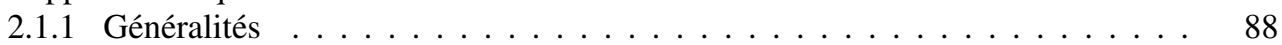

2.1 .2 Le formalisme de van Hove . . . . . . . . . . . . . . . . . . . . . . . . . . . . . . .

2.1 .3 Conséquences expérimentales : . . . . . . . . . . . . . . . . . 92

2.1 .4 Aspects liés à la température de l'échantillon : . . . . . . . . . . . . . . . . . . 92

2.1.5 Modélisation de la dynamique vibrationnelle : . . . . . . . . . . . . 93

2.1.6 Exemples concrets d'utilisation du formalisme de van Hove : calcul de $S_{\text {inc }}(Q, \omega)$ dans le

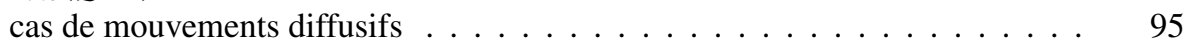

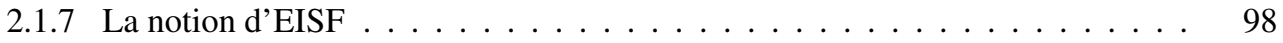

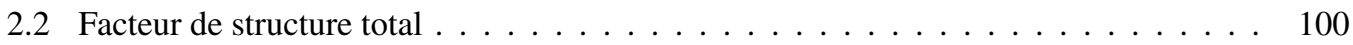

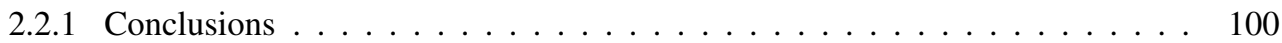

3 Instrumentation $\quad 101$

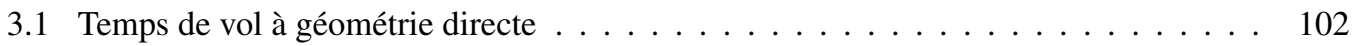

3.1 .1 Les spectromètres à choppers . . . . . . . . . . . . . . . . . . . . . . . . . 102

3.1 .2 Temps de vol à géométrie indirecte . . . . . . . . . . . . . . . . . . . . . . . . . . . . . . . . . . . . . . . . . . . . . . .

3.2 Conséquence des effets de résolution . . . . . . . . . . . . . . . . 105 
4 Une protéine est-elle un verre ?

4.1 Transition dynamique et fonction $\ldots \ldots \ldots \ldots \ldots \ldots$

4.2 Propriétés des verres et des liquides surfondus . . . . . . . . . . . . . . 108

4.3 Un exemple d'approche statistique de la dynamique des protéines : la dynamique du lysozyme . . . . . . . . . . . . . . . . . . . . 109

5 La dynamique de la parvalbumine. Une approche mécanistique 110

6 Conclusion

\section{INTRODUCTION}

Une protéine est un assemblage moléculaire complexe dont l'élément de base est la liaison peptidique. C'est l'enchaînement successif des ces liaisons qui forme "l'ossature" de la protéine. La caractéristique essentielle de cette architecture est la variété des chaînes latérales (Fig.1) qui rend possible toute la palette d'interactions moléculaires: liaisons hydrogènes, interactions de van der Waals, interactions électrostatiques. L'immersion d'un tel édifice moléculaire dans un bain thermique $\left(k_{B} T, k_{B}\right.$ est la constante de Boltzmann et $\mathrm{T}$ la température) génère un spectre étendu d'événements dynamiques allant de la vibration atomique localisée à une dynamique relaxationnelle à grande échelle de toute la structure tertiaire ou quaternaire (Fig.1). Cet article illustre, par quelques exemples précis, comment la diffusion de neutrons permet d'appréhender ces phénomènes.

\section{LA DIFFUSION INCOHÉRENTE INÉLASTIQUE DE NEUTRONS ASPECTS THÉORIQUES ET TECHNIQUES}

\subsection{Rappels théoriques}

\subsubsection{Généralités}

Dans le cadre de la dualité onde-corpuscule, on associe une onde à un neutron. Les faisceaux de neutrons thermiques ${ }^{1}$ mis à la disposition de la communauté scientifique sont conçus pour offrir une gamme de longueur d'onde de l'ordre de grandeur des distances interatomiques. L'interaction d'un faisceau de neutrons avec la matière conduit donc à un phénomène de diffusion, dont l'analyse permet d'accéder à la structure locale de l'échantillon. Une seconde particularité particulièrement intéressante des neutrons thermiques est que leur énergie est de l'ordre de grandeur de nombreuses excitations présentes dans la matière condensée. L'analyse de l'énergie échangée entre le neutron et l'échantillon permet donc d'accéder également à la dynamique du système étudié. Ces études de dynamique privilégient une propriété spécifique de la diffusion des neutrons par la matière: la diffusion inélastique incohérente qui donne accès à la dynamique individuelle des atomes. La diffusion cohérente permet d'accéder aux propriétés collectives (structurales ou dynamiques) du système.

Comme dans toutes les expériences de diffusion de rayonnement, une expérience de diffusion de neutrons consiste à "éclairer" un échantillon par un faisceau de neutrons de vecteur d'onde $\overrightarrow{k_{0}}$ et d'énergie $E_{0}$. Sous l'effet du potentiel d'interaction $V(\vec{r}, t)$ entre le neutron et le système, après passage dans l'échantillon, le rayonnement est caractérisé par le vecteur d'onde $\overrightarrow{k_{1}}$ et l'énergie $E_{1}$ (Fig. 2). L'angle de diffusion, $2 \theta$, est défini comme l'angle entre les vecteurs $\vec{k}_{1}$ et $\vec{k}_{0}$.

L'énergie d'un neutron de vecteur d'onde $\vec{k}$ est:

$$
E=\hbar \omega=\frac{\hbar^{2}|\vec{k}|^{2}}{2 m_{n}}
$$

\footnotetext{
${ }^{1}$ Neutrons thermiques: neutrons dont l'énergie associée est de l'ordre de grandeur de l'agitation thermique à température ambiante, c'est à dire $25 \mathrm{meV}=200 \mathrm{~cm}^{-1}$.
} 

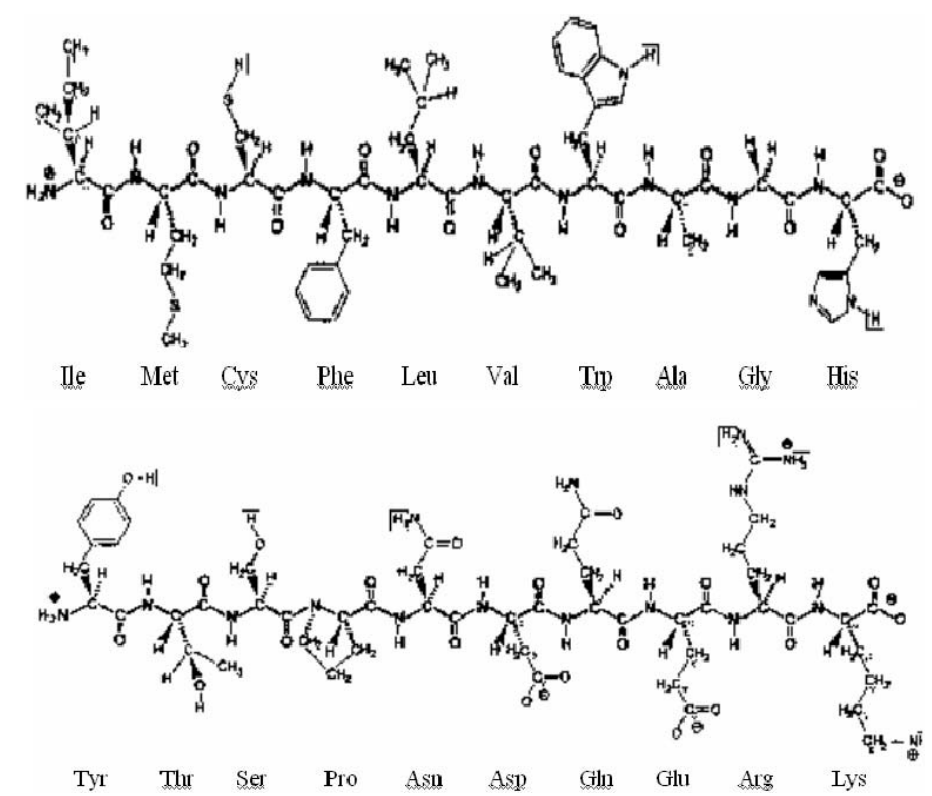

\section{The Rich Dynamics of Hierarchically Organised Biomolecules $2 \pi / \mathrm{Q}$}

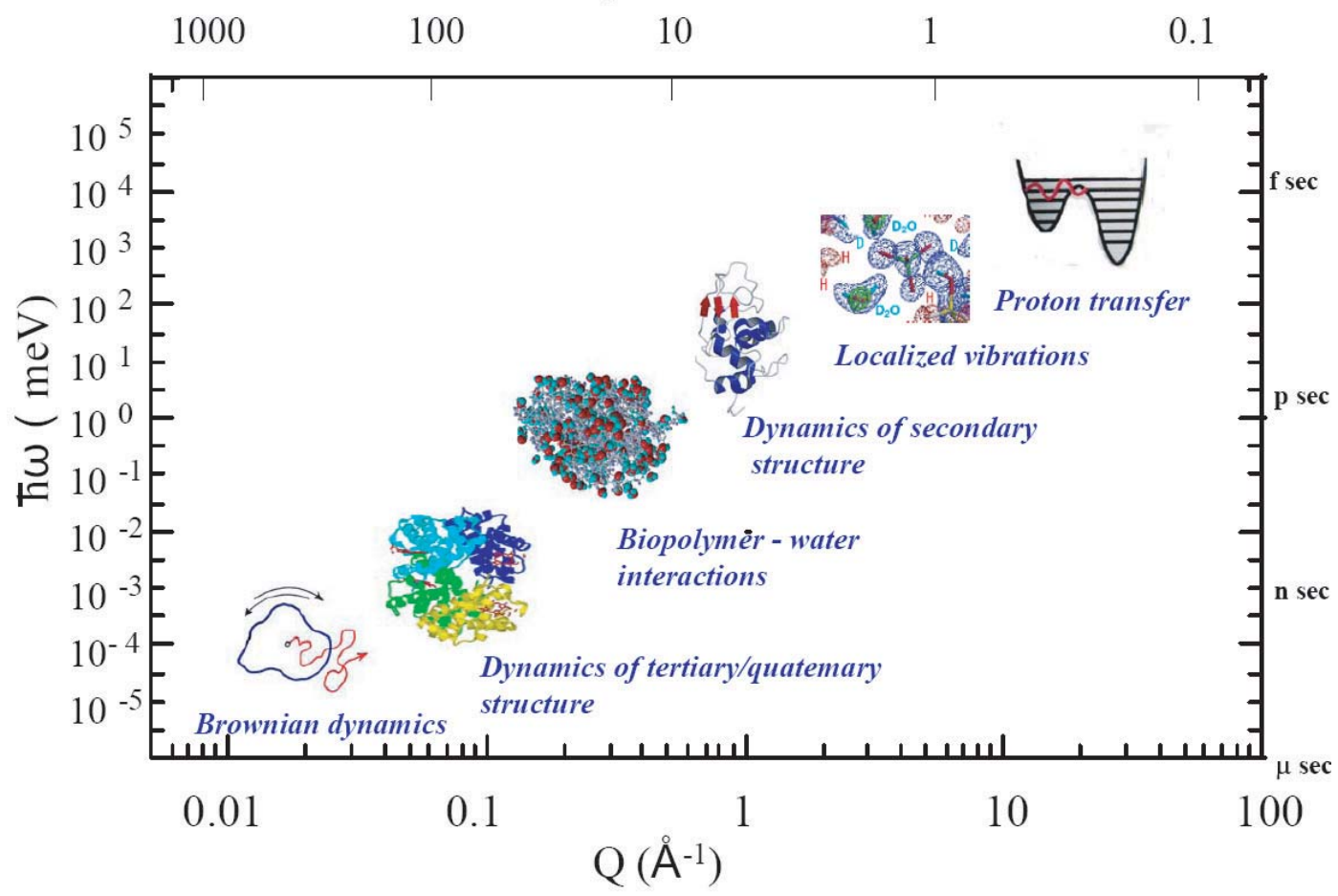

Figure 1. Figure du haut: La totalité des vingt chaînes latérales possibles est reportée, ici à gauche, sur un polypeptide imaginaire où elles sont regroupées en residus hydrophobes (en haut) et hydrophiles (en bas). Figure du bas: La terminologie "dynamique des protéines" regroupe des phénonènes dynamiques aux caractéristiques de temps (échelle de gauche) et d'espace (échelle supérieure) très différentes, dont les phénomènes les plus représentatifs sont illustrés sur le graphe de droite (d'après SHIBATA, JAERI). 

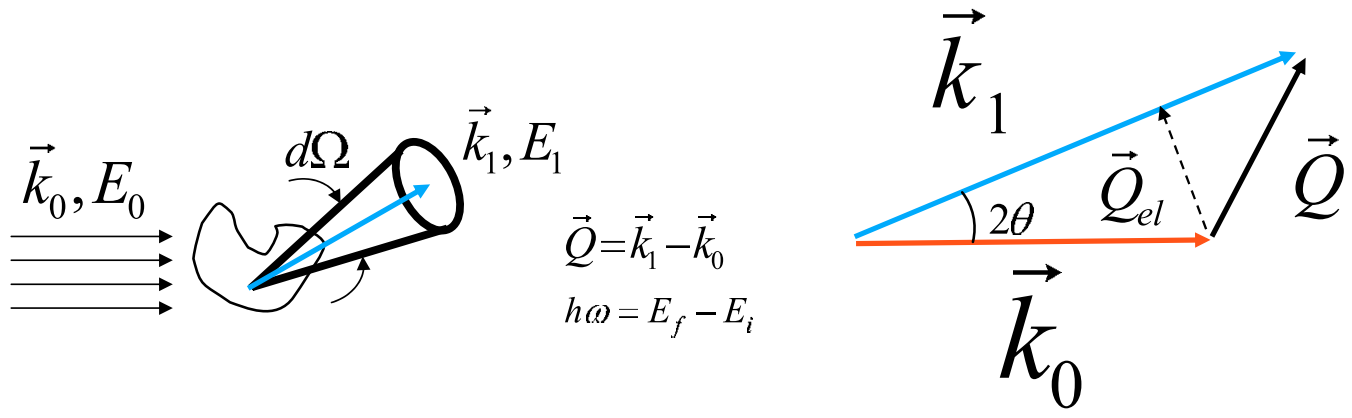

Figure 2. Principe d'une expérience de diffusion de neutrons. Sous l'effet de l'interaction avec l'échantillon, les neutrons incidents subissent une modification de vecteur d'onde $\vec{k}$ et d'énergie $\mathrm{E}$. $\vec{Q}_{e l}=\frac{4 \pi}{\lambda} \sin (\theta) . \vec{u}$, où $\vec{u}$ désigne un vecteur unitaire, est le vecteur de diffusion élastique. Il correspond à un processus de diffusion sans échange d'énergie, i.e. pour lequel $\hbar \omega=0$. Dans le cas général d'une interaction inélastique $(\hbar \omega \neq 0)$, Q est une fonction de $\theta$ et $\omega$. Dans l'espace réciproque i.e. l'espace du détecteur, les deux grandeurs de travail sont $\mathbf{Q}$ et $\omega$. Les deux grandeurs conjuguées de l'espace réel, i.e. l'espace de l'échantillon, sont le temps, t, et l'espace, r. On passe d'un espace à l'autre par transformée de Fourier sur $\mathrm{r}$ et $\mathrm{t}$.

où $m_{n}$ est la masse du neutron et $\hbar$, la constante de Planck divisée par $2 \pi$. Les règles de conservation de l'impulsion et de l'énergie conduisent aux deux relations:

$$
\begin{aligned}
\vec{Q} & =\vec{k}_{1}-\vec{k}_{0} \\
\hbar \omega & =\hbar\left(\omega_{0}-\omega_{1}\right)
\end{aligned}
$$

où $\vec{Q}$ est le vecteur de diffusion et $\hbar \omega$ est l'énergie perdue ou gagnée par le neutron ${ }^{2}$. Dans la suite, par convention, les valeurs négatives de $\omega$ correspondront à une perte d'énergie de l'échantillon, donc à un gain d'énergie du neutron.

Expérimentalement, on mesure la section efficace différentielle de diffusion [1] c'est à dire le nombre de neutrons diffusés, par unité d'angle solide, $d \Omega$, et par unité de transfert d'énergie, $d \omega$. Cette grandeur dépend de l'évolution des positions $\overrightarrow{R_{i}}(t)$ relatives des atomes du système au cours du temps:

$$
\frac{d^{2} \sigma}{d \Omega d \omega}=\frac{1}{2 \pi} \frac{k_{1}}{k_{0}} \sum_{i, j}\left\langle b_{i} b_{j}\right\rangle \int_{-\infty}^{+\infty}\left\langle e^{i \vec{Q} \vec{R}_{i}(0)} e^{-i \vec{Q} \vec{R}_{j}(t)}\right\rangle e^{-i \omega t} d t
$$

Une propriété essentielle de la diffusion de neutrons est la dépendance en spin de l'interaction neutronnoyau et donc des longueurs de diffusion $b_{i}$. L'analyse détaillée des phénomènes associés dépasse le cadre de ce document. Nous dirons simplement que le développement du terme $\left\langle b_{i} b_{j}\right\rangle$ de l'équation 2.3 permet de décomposer l'intensité diffusée en terme de diffusion cohérente et incohérente:

$$
\left(\frac{d^{2} \sigma}{d \Omega d \omega}\right)=\left(\frac{d^{2} \sigma}{d \Omega d \omega}\right)_{c o h}+\left(\frac{d^{2} \sigma}{d \Omega d \omega}\right)_{i n c}
$$

Les relations 2.5 et 2.6 ci-dessous, permettent de clairement distinguer les paramètres inhérents à la technique - la pondération de chacun des termes de l'équation 2.4 par des sections de diffusion de neutrons $\sigma_{c o h}$ et $\sigma_{\text {incoh }}$ - des grandeurs $S(\vec{Q}, \omega)$ et $S_{i n c}(\vec{Q}, \omega)$ qui portent l'information physique

\footnotetext{
${ }^{2}$ Formules usuelles et ordres de grandeurs:

L'énergie d'un neutron de longueur d'onde $\lambda$ est $E(m e V)=\frac{81.805}{\lambda\left(\AA \AA^{2}\right)^{2}}$.

Temps de parcourt, $\tau$, d'une distance $\mathrm{D}$, pour un neutron de longueur d'onde $\lambda: \tau(\mu \mathrm{s})=252.778 * D(m) * \lambda(\AA)$ : un neutron de $5 \AA$ parcourt 1 mètre en $1.26 \mathrm{~ms}$.
} 
sur le système:

$$
\begin{aligned}
& \left(\frac{d^{2} \sigma}{d \Omega d \omega}\right)_{c o h}=\frac{k_{1}}{k_{0}} \frac{\sigma_{c o h}}{4 \pi} S(\vec{Q}, \omega) \\
& \left(\frac{d^{2} \sigma}{d \Omega d \omega}\right)_{i n c}=\frac{k_{1}}{k_{0}} \frac{\sigma_{i n c}}{4 \pi} S_{i n c}(\vec{Q}, \omega)
\end{aligned}
$$

$S(\vec{Q}, \omega)$ et $S_{\text {inc }}(\vec{Q}, \omega)$ sont appelés facteurs de structure dynamiques respectivement cohérent et incohérent.

La distinction la plus directe entre diffusion cohérente et incohérente se matérialise par la dépendance en $\mathrm{Q}$ des intégrales en énergie des facteurs de structure dynamiques:

$$
\begin{aligned}
\int S(\vec{Q}, \omega) d \omega & =S(Q) \\
\int S_{\text {inc }}(\vec{Q}, \omega) d \omega & =1
\end{aligned}
$$

Contrairement au facteur de structure cohérent $\mathrm{S}(\mathrm{Q})$ (Eq. 2.7) qui se présente comme une fonction structurée en Q (pics de Bragg, halo dans les liquides etc...), l'intensité incohérente intégrée est parfaitement indépendante de Q (Eq. 2.8). Dans les expériences de diffraction ou de diffusion aux petits angles, elle est donc souvent qualifiée de "bruit de fond" incohérent et on cherche à minimiser sa contribution. Comme nous tenterons de l'illustrer dans ce qui suit bien plus qu'un bruit de fond, c'est une grandeur dont la dépendance en énergie est porteuse d'une information très riche.

\subsubsection{Le formalisme de van Hove}

Considérons une assemblée de particules. Soit $\mathrm{G}(\mathrm{r}, \mathrm{t})$ une fonction de corrélation qui, considérant une particule $P$ à l'origine de l'espace $r=0$ à $\mathrm{t}=0$ donne la probabilité de trouver une particule $P$ ' à une distance $r$ au temps t. On peut scinder G(r,t) en une contribution d'autocorrélation ("self"), $G_{s}(r, t)$, et une contribution de corrélation de paire ("distinct"), $G_{d}(r, t)$ :

$$
G(r, t)=G_{s}(r, t)+G_{d}(r, t)
$$

$G_{s}(r, t)$ donne la probabilité de trouver la particule initiale $P$ à r au temps t, tandis que $G_{d}(r, t)$ donne la probabilité d'y trouver une particule $P$ ' autre que $P$.

Le formalisme de van Hove [2] opère un lien direct entre les fonctions de corrélations ci-dessus et l'intensité diffusée:

$$
\begin{aligned}
S(\vec{Q}, \omega) & =\frac{1}{2 \pi} \int G(\vec{r}, t) e^{i(\vec{Q} \vec{r}-\omega t)} d \vec{r} d t \\
S_{i n c}(\vec{Q}, \omega) & =\frac{1}{2 \pi} \int G_{s}(\vec{r}, t) e^{i(\vec{Q} \vec{r}-\omega t)} d \vec{r} d t
\end{aligned}
$$

- L'intensité cohérente étant en partie reliée au terme "distinct" $G_{d}(r, t)$ porte une information collective. Comme on l'a vu (Eq. 2.7), l'analyse de l'intensité intégrée en énergie diffusée par un système cohérent permet donc d'accéder à la structure du système:

$$
S(Q)=\int S(Q, \omega) d \omega=G(r, t=0)
$$

Si on considère maintenant la dépendance en énergie de l'intensité cohérente, il est possible d'accéder aux modes collectifs (phonons). 
- L'intensité incohérente du système n'est reliée qu'à la partie "self" $G_{s}(r, t)$ et ne porte donc qu'une information individuelle. Elle ne permet donc pas la détermination de structures (Eq. 2.8). Selon le domaine en énergie associé à la diffusion incohérente on définit:

* la diffusion incohérente élastique qui permet d'accéder à des informations sur la localisation des atomes (écarts quadratiques moyens associés aux vibrations, espaces de confinement...).

* la diffusion incohérente quasi-élastique (i.e. faiblement inélastique dans la zone $\hbar \omega \approx 0$ ) qui permet la mesure de coefficients de self-diffusion, temps de résidence...

* la diffusion incohérente inélastique Il s'agit de spectroscopie vibrationnelle. Elle permet de déterminer des densités d'états vibrationnels i.e. la distribution en énergie des modes de vibration du système.

Chacun de ces termes est évoqué en détails dans les paragraphes suivants.

Cohérence et incohérence ne sont pas des propriétés intrinsèques de la particule neutron: un neutron n'est ni cohérent ni incohérent et dans tous les cas, les spectromètres détectent des neutrons, pas des "neutrons cohérents" ou des "neutrons incohérents". Cohérence ou incohérence sont dictées par la composition isotopique du système. Des dispositifs spécifiques (polariseurs et analyseurs) permettent cependant de n'accéder qu'à la fraction cohérente ou incohérente de l'intensité diffusée.

\subsubsection{Conséquences expérimentales:}

Les notions de cohérence et d'incohérence permettent de faire expérimentalement la distinction entre comportements collectifs et individuels. Le tableau 1 regroupe les valeurs des sections efficaces de diffusion cohérente et incohérente d'espèces chimiques ou d'isotopes qui constituent l'essentiel des atomes d'échantillons biologiques.

Table 1. Longueurs de diffusion cohérentes et sections efficaces de diffusion de différents atomes. Les sections efficaces d'absorption, $\sigma_{a b s}$, sont données pour une longueur d'onde incidente de $1.8 \AA$ A [3].

\begin{tabular}{|c|c|c|c|c|}
\hline \hline Espèce & $\begin{array}{c}b_{\text {coh }} \\
(\mathrm{fm})\end{array}$ & $\begin{array}{c}\sigma_{\text {coh }} \\
(\text { barn })\end{array}$ & $\begin{array}{c}\sigma_{\text {inc }} \\
(\text { barn })\end{array}$ & $\begin{array}{c}\sigma_{a b s} \\
(\text { barn })\end{array}$ \\
\hline $\mathrm{C}$ & 6.64 & 5.55 & 0.001 & 0.0035 \\
$\mathrm{~N}$ & 9.36 & 11.01 & 0.50 & 1.90 \\
$\mathrm{O}$ & 5.803 & 4.232 & 0 & 0.000191 \\
$\mathrm{~S}$ & 2.847 & 1.0188 & 0.007 & 0.53 \\
$\mathrm{H}$ & -3.7406 & 1.7583 & 80.27 & 0.3326 \\
$\mathrm{D}$ & 6.671 & 5.592 & 2.05 & 0.000519 \\
\hline
\end{tabular}

On constate que l'atome d'hydrogène a une section efficace de diffusion incohérente très importante, 40 fois plus élevée que celle de son isotope, le deutérium. Dans les composés hydrogénés, la contribution incohérente de l'hydrogène domine donc largement les contributions cohérente et incohérente de tous les autres atomes du composé. Le facteur de structure dynamique incohérent peut donc être directement relié à la dynamique individuelle des atomes d'hydrogène de l'échantillon.

\subsubsection{Aspects liés à la température de l'échantillon:}

Considérons un neutron d'énergie incidente $\hbar \omega_{0}$ interagissant avec une assemblée d'oscillateurs harmoniques à température T, i.e. d'énergie moyenne $E=k_{B} \cdot T$.

- Si $k_{B} T \gg \hbar \omega_{0}$, de nombreux oscillateurs sont dans un état excité et le neutron incident a une forte probabilité de gagner de l'énergie lors de l'interaction. Dans ce cas, le facteur limitant l'observation 
d'une transition à un transfert d'énergie donné est le peuplement du niveau correspondant, qui dépend de la température de l'échantillon. La probabilité que le neutron perde de l'énergie n'est pas nulle, cependant, le neutron ne pouvant perdre plus d'énergie qu'il n'en a, le transfert maximum d'énergie est limité à $+\hbar \omega_{0}$.

- Si $k_{B} T \ll \hbar \omega_{0}$, les niveaux excités ne sont peu ou pas peuplés; c'est grace à l'énergie du neutron qu'ils vont être peuplés. Dans ce cas, c'est l'énergie initiale du neutron qui limitera le transfert d'énergie maximal observable et non la température de l'échantillon.

On considère le facteur de population de Bose: $n=1 /\left(e^{-\hbar \omega / k_{B} T}-1\right)$. Les considérations ci-dessus expliquent qu'à vecteur de diffusion $\mathrm{Q}$ donné, l'intensité observée soit fonction de $\omega$ mais aussi de la température, T, de l'échantillon:

$$
\left(\frac{d^{2} \sigma}{d \Omega d \omega}\right)_{i n c}=\frac{k_{1}}{k_{0}} \frac{\sigma_{i n c}}{4 \pi} \cdot n_{B}(\omega, T) \cdot S_{i n c}(\vec{Q}, \omega)
$$

avec $n_{B}(\omega, T)=n+1$ si $\omega<0$ et $n_{B}(\omega, T)=n$ si $\omega>0$. La même relation s'applique au cas de la diffusion cohérente. On remarquera que $2 n+1=\operatorname{coth}\left(\hbar \omega / 2 k_{B} T\right)$. Ces considérations sur le rapport $\hbar \omega_{0} / k_{B} T$ ont d'importantes conséquences quant au choix des conditions expérimentales et du type de spectromètre permettant d'optimiser l'observation d'une excitation (cf. sections 3.1.1 et 3.1.2).

\subsubsection{Modélisation de la dynamique vibrationnelle :}

La fonction des protéines est généralement corrélée à des mouvements de grandes amplitudes de domaines. C'est par exemple le cas du lysozyme (14 kDa, 129 résidus) dont la fonction est la catalyse de l'hydrolyse des parois bactériennes (polysaccharides). Cette fonction biologique est subordonnée à un mouvement de charnière des deux domaines de l'enzyme qui viennent mettre le site actif au contact de deux liaisons glycosidiques séparant deux cycles successifs de la chaîne de polysaccharide. L'analyse en modes normaux est une méthode numérique permettant, connaissant la structure de la macromolécule, de calculer les fréquences et les amplitudes des mouvements collectifs auxquels sont soumis les atomes du système: chaque atome est supposé soumis à des vibrations autour de sa position d'équilibre et on introduit des interactions entre ces oscillateurs. Ce couplage peut provoquer des mouvements collectifs que l'on peut décrire comme étant la résultante des vibrations d'oscillateurs harmoniques indépendants, les modes normaux de vibration. Dans le cas de petites protéines globulaires comme le lysozyme, les fréquences des modes propres s'étalent de quelques fractions à quelques centaines de $\mathrm{cm}^{-1}$ [8]. En théorie, la diffusion inélastique de neutrons permet d'accéder à ces information de façon expérimentale. La fonction de diffusion intermédiaire d'une assemblée d'atomes vibrant harmoniquement s'écrit:

$$
I(\vec{Q}, t)=\sum_{i, j} e^{-\left\langle\left(\vec{Q} \vec{u}_{i}\right)^{2}\right\rangle} e^{\left\langle\vec{Q} \vec{u}_{i}(0) \vec{Q} \vec{u}_{j}(t)\right\rangle}
$$

On ne s'intéresse qu'à la fonction d'autocorrélation, on ne prend donc en compte que le cas ${ }^{3} \mathrm{i}=\mathrm{j}$ :

$$
I_{s}(\vec{Q}, t)=\sum_{i} e^{-\left\langle\left(\vec{Q} \vec{u}_{i}\right)^{2}\right\rangle} e^{\left\langle\vec{Q} \vec{u}_{i}(0) \vec{Q} \vec{u}_{i}(t)\right\rangle}
$$

On peut décrire le mouvement vibrationnel d'un atome appartenant à une molécule composée de $\mathrm{N}$ atomes comme la combinaison de $3 \mathrm{~N}-6$ modes normaux. Chaque mode est caractérisé par une pulsation $\omega_{\lambda}$ et $\mathrm{N}$ vecteurs propres $\vec{C}_{i}^{\lambda}$. Ils donnent l'amplitude relative et la direction du déplacement de chaque

\footnotetext{
${ }^{3}$ Le cas $i \neq j$ rend compte de mouvements vibrationnels corrélés: les phonons.
} 
atome selon le mode $\lambda$. La trajectoire d'un atome i de masse $m_{i}$ est alors:

$$
\vec{u}_{i}(t)=\sum_{\lambda=1}^{3 N-6} A_{\lambda} \frac{\vec{C}_{i}^{\lambda}}{\sqrt{m_{i}}} \cos \left(\omega_{\lambda} t+\Phi_{\lambda}\right)
$$

où $A_{\lambda}$ et $\Phi_{\lambda}$ sont l'amplitude et la phase du $n^{i \grave{m} e}$ mode normal. Les vecteurs propres forment une base orthonormale: $\sum_{i=1}^{N} \vec{C}_{i}^{\lambda} \vec{C}_{i}^{\lambda^{\prime}}=\delta_{\lambda \lambda^{\prime}}$. Pour un système à l'équilibre thermique, à la température T, telle que $\hbar \omega \ll k_{B} T$, le théorème d'équipartition conduit à: $A_{\lambda}=\sqrt{\frac{k_{B} T}{\omega_{\lambda}^{2}}}$. En introduisant ce résultat dans l'équation 2.14:

$$
I_{s}(\vec{Q}, t)=\sum_{i} e^{-2 W_{i}(\vec{Q})}\left\{1+\sum_{\lambda} \frac{\left|\vec{Q} \vec{C}_{i}^{\lambda}\right|^{2}}{2 m_{i}}\left\langle\cos \left(\Phi_{\lambda}\right) \cos \left(\omega_{\lambda} t+\Phi_{\lambda}\right)\right\rangle\right\}
$$

où:

$$
W_{i}(\vec{Q})=\sum_{\lambda=1}^{3 N-6} \frac{k_{B} T}{2 m_{i}} \frac{\left|\vec{Q} \vec{C}_{i}^{\lambda}\right|^{2}}{\omega_{\lambda}} \operatorname{coth}\left(\frac{\hbar \omega}{2 k_{B} T}\right)
$$

$e^{-2 W_{i}(\vec{Q})}$ est le facteur de Debye-Waller. Ce terme mesure l'extension spatiale, dans la direction $\vec{Q}$, des mouvements dus à l'ensemble des modes. On obtient le facteur de structure dynamique incohérent par transformée de Fourier:

$$
\begin{aligned}
& S_{\mathrm{inc}}^{\mathrm{vib}}(\vec{Q}, \omega)= \\
& \sum_{i} e^{-2 W_{i}(\vec{Q})}\left\{\delta(\omega)+\sum_{\lambda} \frac{k_{B} T}{2 m_{i}} \frac{\left|\vec{Q} \vec{C}_{i}^{\lambda}\right|^{2}}{\omega_{\lambda}}\left[n_{B}(\omega, T) \delta\left(\omega-\omega_{\lambda}\right)+\left(n_{B}(\omega, T)+1\right) \delta\left(\omega+\omega_{\lambda}\right)\right]\right\}
\end{aligned}
$$

où $n_{B}(\omega, T)$ est le facteur de bose ( $\left.\operatorname{cf} 2.1 .4\right)$. A ce terme de vibrations internes, il est nécessaire de rajouter des termes provenant de la vibration du réseau. Sous l'influence de ces modes, la molécule entière peut être soumise à des mouvements de libration, de translation ou de rotation. Cependant, ce terme de vibration du réseau présente peu d'évolution en énergie. Dans la zone quasi-élastique du spectre, il peut être assimilé à un bruit de fond plat dépendant de Q ( Cf. référence [5], pages 65 et 67). Par moyenne de poudre l'expression $\left\langle\left|\vec{C}_{i}^{\lambda} \vec{Q}\right|^{2}\right\rangle$ se simplifie: $\left\langle\left|\vec{C}_{i}^{\lambda} \vec{Q}\right|^{2}\right\rangle=\frac{1}{3} Q^{2}\left|\vec{C}_{i}^{\lambda}\right|^{2}$. De l'expression 2.19, on déduit:

$$
\begin{aligned}
\lim _{Q \rightarrow 0} S_{i n c}^{v i b}(Q, \omega) \frac{6 \omega}{\hbar Q^{2}}\left(n_{B}(\omega, T)+1\right) & =\sum_{\lambda=1}^{3 N-6} \sum_{i=1}^{N} \frac{\left|\vec{C}_{i}^{\lambda}\right|^{2}}{m_{i}} \delta\left(\omega+\omega_{\lambda}\right) \\
& =G(\omega)
\end{aligned}
$$

La fonction $G(\omega)$ est la densité d'états pondérée. Elle permet d'estimer la vraie densité d'états vibrationnels:

$$
g(\omega)=\sum_{\lambda=1}^{3 N-6} \delta\left(\omega+\omega_{\lambda}\right)
$$

Contrairement aux spectroscopies infra-rouge ou raman, il n'y a pas de règles de sélection en diffusion inélastique de neutrons. Toute les transitions accessibles dans la gamme d'énergie où est réalisée l'expérience sont permises. On peut donc accéder à une vraie densité d'états pondérée. Notons cependant que l'intensité des excitations dépend fortement de la température du système.

On appelle densité d'états vibrationnels, $g(\omega)$, la fonction de distibution des fréquences vibrationnelles (modes propres, fréquences de résonance... ) d'un système. Considérons une assemblée 
de $\mathrm{N}$ oscillateurs harmoniques de masse $\mathrm{M}$ soumis à divers modes inélastiques isotropes en trois dimensions, de densité d'états $g(\omega)$. A une température T, l'intensité diffusée de façon élastique est proportionnelle à:

$$
S(Q, \omega=0)=e^{-Q^{2}\langle u\rangle^{2} / 3} . \delta(\omega) \quad \text { avec } \quad\langle u\rangle^{2}=\frac{\hbar}{6 M N} \int \frac{g(w)}{w} \operatorname{coth}\left(\frac{\hbar \omega}{2 k_{B} T}\right) d w
$$

Pour $\hbar \omega \gg 2 k_{B} T$, à haute température par exemple, $\operatorname{coth}(x) \simeq \frac{1}{x}$ et l'équation 2.21 se simplifie:

$$
\langle u\rangle^{2}=\frac{3 k_{b} T}{M} \int \frac{g(w)}{w^{2}} d w
$$

Dans un système purement hamonique les modes de vibration, donc $g(\omega)$, sont rigoureusement indépendants de la température. Celle-ci règle cependant le peuplement des différents nivaux de vibration, par l'intermédiaire du facteur de population de Bose $n(\omega)$ (équivalent à la statistique de MaxwellBoltzmann à haute température) et c'est par ce biais qu'elle contrôle l'intensité diffusée.

L'équation 2.21 montre que les modes vibrationnels (vibration des atomes autour de leur position d'équilibre) induisent une chute gaussienne de l'intensité diffusée à grands Q - il s'agit de l'effet DebyeWaller, célèbre également en diffraction des rayons $\mathrm{X}$ - et que ce phénomène est d'autant plus prononcé que la température est élevée.

L'intensité diffusée dans la zone inélastique du spectre donne accès à $g(\omega)$ (Eq. 2.19). D'autre part, selon l'équation 2.21, un ajustement libéaire de $\ln (S(Q, \omega \approx 0))$ vs $Q^{2}$ permet de déterminer l'extension spatiale, $\langle u\rangle^{2}$, des vibrations. Précisons cependant, qu'un solide étant essentiellement statique, les neutrons subissent une diffusion principalement élastique et les bandes inelastiques sont beaucoup moins intenses que le pic élastique (rapport de l'ordre de un à cent). La mesure fine de la densité d'états vibrationnels, $g(\omega)$, demande un excellent rapport signal sur bruit.

Un écart à la linéarité de $\langle u\rangle^{2}$ vs T est caractéristique de l'apparition d'une dynamique anharmonique et/ou de l'apparition de modes dynamiques non vibrationnels, diffusionnels par exemple. Une généralisation du formalisme ci-dessus, permettant de prendre en compte de telles contributions souvent présentes dans les systèmes biologiques, est apportée en conclusion de ce chapitre au paragraphe 2.2.1.

Pour être fonctionnelle une protéine doit adopter un certain degré de flexibilité (voir 4.1 ), à toutes les échelles, si bien que les modes de grandes amplitudes et faible fréquence liés à la fonction, sont expérimentalement masqués par des contributions dynamiques, vibrationnelles et/ou diffusionnelles des chaînes latérales. Du point de vue des modes normaux, il s'agit là de contributions "parasites". Il faut cependant garder à l'esprit que cette dynamique rapide mettant en jeu des mouvements de faibles amplitudes est probablement la source des mouvements lents $(\mu \mathrm{s}-\mathrm{ms})$ de grandes amplitudes liés à la fonction. Cette approche hiérarchique a été en particulier développée par Frauenfelder et collaborateurs [16]. La section qui suit décrit comment modéliser cette dynamique relaxationnelle rapide.

\subsubsection{Exemples concrets d'utilisation du formalisme de van Hove : calcul de $S_{\text {inc }}(Q, \omega)$ dans le cas de mouvements diffusifs}

Diffusion aléatoire à longue distance (loi de Fick). Le mécanisme de diffusion isotrope d'une particule peut être décrit comme le phénomène cyclique suivant: sous l'action d'un choc, avec une autre particule par exemple, la particule est mise en mouvement et parcourt un trajet $\vec{r}_{\text {elem }}$ dans l'espace en un temps $\tau$. A cet instant, un nouvel événement modifie la vitesse et la direction de la particule et l'histoire initiale de la particule (position, vitesse, direction) est oubliée. On montre [5] que, si l'on se place dans des conditions où l'on observe le système à une échelle grande devant $\vec{r}_{\text {elem }}$ et à un temps grand devant $\tau$, la probabilité de trouver la particule en un point $\vec{r}$ de l'espace au temps t obéit à la relation:

$$
\frac{\partial G_{s}(r, t)}{\partial t}=D_{t} \nabla^{2} G_{s}(r, t)
$$




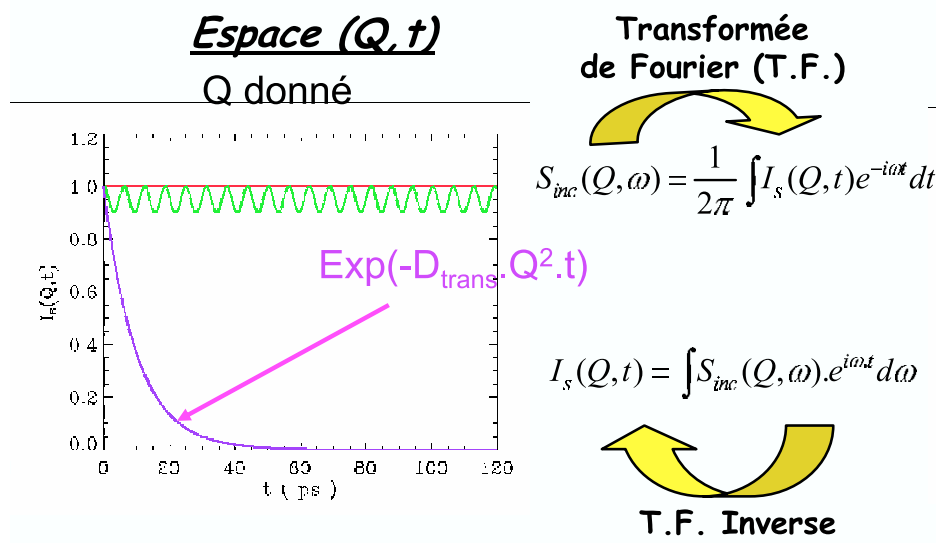

Espace $(Q, \omega)$

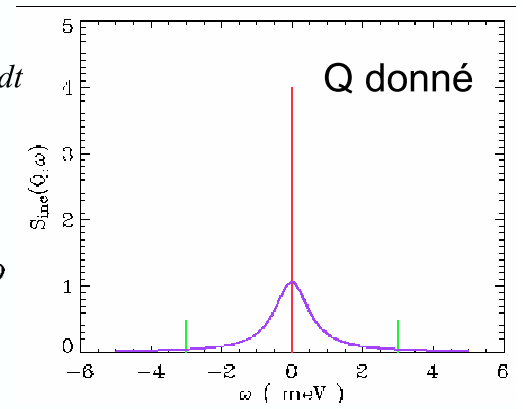

Figure 3. Représentation schématique de l'allure des spectres en énergie de différents modes dynamiques. Considérons la fonction d'autocorrélation $\mathrm{I}(\mathrm{Q}, \mathrm{t})$ des positions d'une particule dans l'espace $(\mathrm{Q}, \mathrm{t})$. On aboutit à la représentation dans l'espace de mesure $(\mathrm{Q}, \omega)$, par transformée de Fourier sur le temps de la fonction $\mathrm{I}(\mathrm{Q}, \mathrm{t})$. Par définition d'une fonction d'autocorrélation, quelle que soit la dynamique de la particule, on connaît sa position à $\mathrm{t}=0$. En terme de probabilité cette certitude se traduit par $\mathrm{I}(\mathrm{Q}, \mathrm{t}=0)=1$. Si la particule est immobile, quel que soit $t$, on connaitra sa position i.e. $I(Q, t)=1$ (cf. courbe rouge). La transformée de Fourier en temps de cette droite horizontale est un dirac en énergie à $\hbar \omega=0$. Ceci correspond physiquement à une situation où les neutrons n'échangent aucune énergie avec la particule. On parle de diffusion élastique. Supposons maintenant que la particule subisse un mouvement vibrationnel de fréquence propre $\hbar \omega_{0}$. La fonction d'autocorrélation I(Q,t) est une fonction sinusoïdale du temps. Sa transformée de Fourrier se matérialise par un dirac élastique encadré par deux diracs centrés à $\hbar \omega_{0}$ et $-\hbar \omega_{0}$. Ces deux diracs de la zone inélastique du spectre $(\hbar \omega \neq 0)$ représentent la plus simple expression de $g(\omega)$, la densité d'états vibrationnels. Dans le cas d'une particule opérant une diffusion translationnelle à longue distance (c.f. paragraphe 2.1.6), I(Q,t) prend une forme exponentielle (Eq. 2.25). Le facteur de structure dynamique correspondant est une Lorentzienne centrée en $\hbar \omega=0$ (Eq. 2.26).

où $D_{t}$ est le coefficient de diffusion translationnel. Avec comme conditions aux limites $G_{s}(Q, 0)=$ $\delta(r)$ et $G_{s}(r, \infty)=0$, la solution de l'équation différentielle 2.23 est:

$$
G(r, t)=\frac{1}{\left(4 \pi D_{t} t\right)^{\frac{3}{2}}} e^{-\frac{r^{2}}{4 D_{t} t}}
$$

Physiquement, cette relation signifie que la probabilité de connaître la position de la particule à une distance $r$ de sa position d'origine est une fonction gaussienne de l'espace (r), dont le carré de l'écart type (quantifiant "l'étalement" de la courbe) est directement proportionnel au temps. Le passage dans l'espace réciproque se fait par transformée de Fourier sur l'espace:

$$
I(Q, t)=e^{-D_{t} \cdot Q^{2} \cdot t}
$$

On obtient le facteur de structure dynamique incohérent correspondant par transformée de Fourier sur le temps:

$$
S_{i n c}(\vec{Q}, \omega)=\frac{1}{\pi} \frac{D_{t} Q^{2}}{\left(D_{t} Q^{2}\right)^{2}+\omega^{2}}
$$

$S_{\text {inc }}(Q, \omega)$ est donc une lorentzienne en énergie d'intensité 1 (i.e. $\int_{-\infty}^{+\infty} S_{i n c}(Q, \omega)=1$ ), de demi-largeur à mi-hauteur $D_{t} Q^{2}$. 

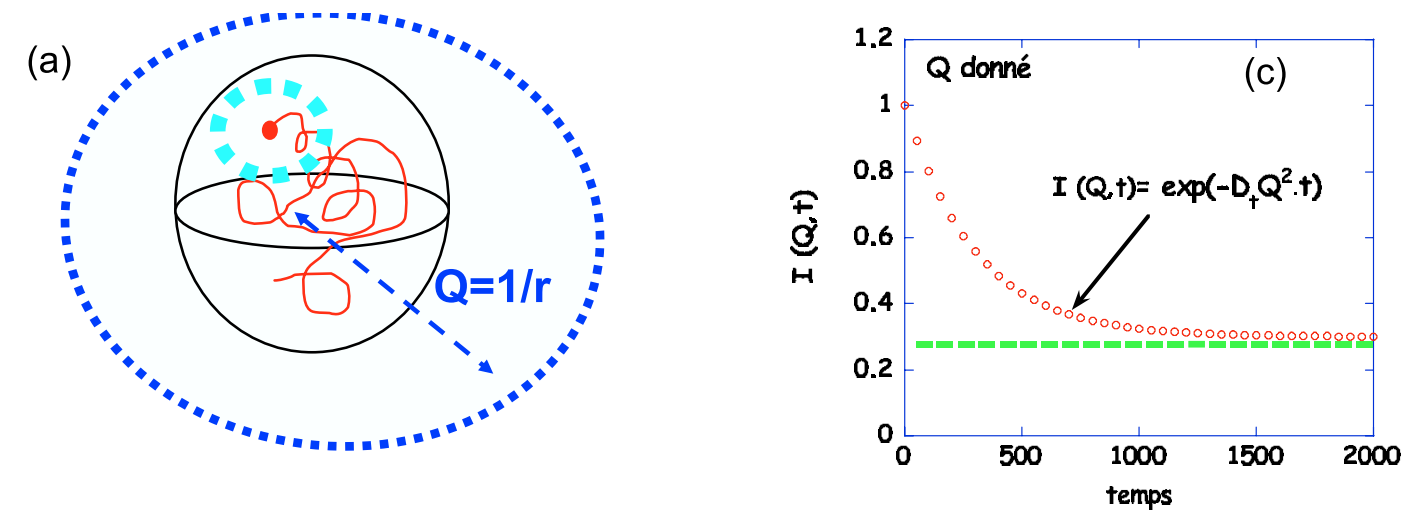

(b)
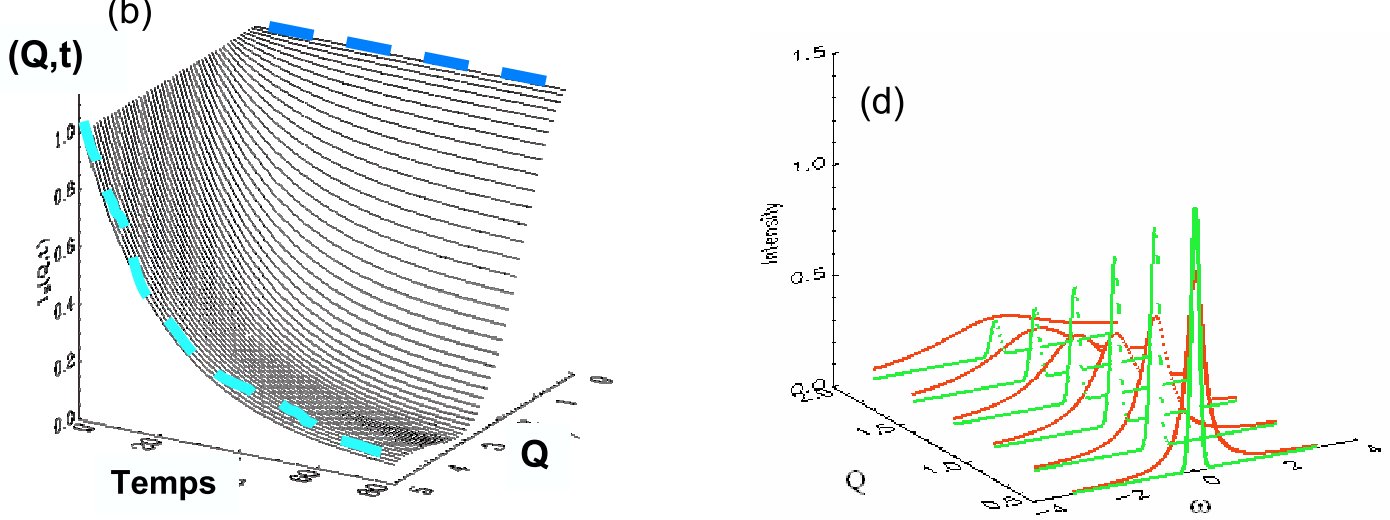

Figure 4. Facteur de Structure Elastique Incohérent: EISF. Considérons une particule (point rouge) soumise à un mouvement de diffusion (trajectoire en rouge) dans un espace de confinement (sphère noire). (b) Représentation de l'évolution de la fonction intermédiaire de diffusion $I_{s}(Q, t)$ correspondante. Chaque courbe représente l'évolution en fonction du temps de $I_{s}(Q, t)$, à une valeur de $\mathrm{Q}$ donnée, i.e. à l'échelle à laquelle on observe le système. Quelque soit cette échelle, à t $=0$, par définition même d'une fonction d'autocorélation, on connaît la position de la particule: $I_{s}(Q, t=0)=1$. A temps plus long, (i) si on regarde le système à une échelle très locale (pointillés bleu clair en (a)) i.e. à grand $\mathrm{Q}$, la particule diffuse et on perd rapidement l'information sur sa position; on arrive vite à $I_{s}(Q, t=0)=0$ (pointillés bleu clair en (b)). (ii) si on observe le système de loin i.e. à une échelle bien plus grande que la taille de la cage de confinement (pointillés bleus en (a), $1 / \mathrm{r}$ est grand donc $\mathrm{Q}$ est petit), à tout instant on connaît la position de la particule: elle est dans la cage de confinement donc $I_{s}(Q, t) \approx 1$ (pointillés bleus en $(\mathrm{b})$ )).

c) Entre ces deux échelles extrêmes d'observation, $I_{s}(Q, t)$ adopte un comportement intermédiaire, caractérisé par la présence d'un plateau indépendant du temps (en vert). Le lieu des points à $I_{s}(Q, t=\infty)$ n'est donc une fonction que de la seule variable Q: ce n'est autre que le facteur de forme (Facteur de structure) de l'espace de confinement. La contribution temporelle, ici de la forme Eq. 2.25 puisque la particule est soumise à un mouvement de diffusion, porte l'information sur la dynamique du système. d)Le facteur de structure élastique incohérent $S_{\text {inc }}(Q, \omega)$ effectivement observé sur un spectromètre en temps de vol s'obtient par transformée de Fourier sur le temps: à chaque valeur de Q, le plateau et l'exponentielle de l'espace $(\mathrm{Q}, \mathrm{t})$ de c) conduisent respectivement (cf. Fig. 3) à un pic élastique d'intensité $I_{E l}=I_{s}(Q, t=\infty)$ et un élargissement quasiélastique d'intensité $\left(I_{Q u a s i}=1-I_{s}(Q, t=\infty)\right)$. Dans l'espace $(\mathrm{Q}, \mathrm{t})$ à chaque $\mathrm{Q}$, le plateau est caractérisé par la fraction élastique du signal. Il en est donc de même dans l'espace $(Q, \omega): E I S F=I_{E l} /\left(I_{E l}+I_{\text {Quasi }}\right)$.

La représentation de la demi-largeur à mi-hauteur ${ }^{4}$ de la lorentzienne quasi-élastique en fonction de $Q^{2}$ est une droite de pente $D_{t}$. On parle de loi en $D Q^{2}$.

\footnotetext{
${ }^{4}$ Half Width at Half Maximum (HWHM) en anglais.
} 
Diffusion par sauts. Dans la situation où la gamme $(Q, \omega)$ utilisée permet d'accéder à des phénomènes aux caractéristiques spatiales et temporelles du même ordre de grandeur que ceux du processus élémentaire conduisant à la diffusion, les conditions qui nous ont conduits à écrire l'équation 2.23 ne sont plus vérifiées. C'est le cas lorsqu' on travaille à grand vecteur de diffusion. Dans le modèle de diffusion par sauts [5], on suppose qu'entre deux événements conduisant à la diffusion, la particule reste sur un site donné pendant un temps $\tau_{0}$, très long devant la durée d'un saut $\tau$. Dans ce cas, le facteur de structure dynamique s'écrit:

$$
S_{\text {inc }}(Q, \omega)=\frac{1}{\pi} \frac{f(Q)}{f(Q)^{2}+\omega^{2}}
$$

$S_{\text {inc }}(Q, \omega)$ est donc une lorentzienne en énergie d'intensité 1 , de demi-largeur à mi-hauteur $f(Q)$ :

$$
f(Q)=\frac{D_{t} Q^{2}}{1+D_{t} Q^{2} \tau_{0}}
$$

La représentation de la demi-largeur à mi-hauteur de l'élargissement quasi-élastique est similaire à la loi en $D Q^{2}$ pour $\mathrm{Q}$ petit. Pour les grandes valeurs de $\mathrm{Q}, \mathrm{f}(\mathrm{Q})$ s'éloigne du comportement linéaire en $\mathrm{Q}$ pour tendre vers une valeur asymptotique égale à l'inverse du temps de résidence $\tau_{0}$.

\subsubsection{La notion d'EISF}

La dynamique individuelle des atomes d'un système est décrite par la fonction d'autocorrélation de van Hove. A $\mathrm{t}=0$ :

$$
G_{s}(\vec{r}, 0)=\delta(\vec{r})
$$

La contribution "self" de la fonction intermédiaire de diffusion est obtenue par inversion de Fourier de la partie self de la fonction de corrélation de van Hove:

$$
I_{s}(\vec{Q}, t)=\int G_{s}(r, t) e^{i \vec{Q} r} d \vec{r}
$$

Imaginons qu'un atome du système que nous étudions soit soumis à une perturbation à $t=0$. Lorsque le système a atteint son état d'équilibre, il n'y a aucune corrélation entre les positions des atomes diffuseurs à $\mathrm{t}=0$ et $t=\infty$. Autrement dit, après avoir réagi à la perturbation imposée à $\mathrm{t}=0$, le système relaxe vers son état d'équilibre initial [4]:

$$
\begin{aligned}
I_{s}(\vec{Q}, t=+\infty) & =\left\langle e^{-i \vec{Q} \vec{r}(0)} e^{-i \vec{Q} \vec{r}(t=+\infty)}\right\rangle \\
& =\left\langle e^{-i \vec{Q} \vec{r}(0)}\right\rangle\left\langle e^{-i \vec{Q} \vec{r}(t=+\infty)}\right\rangle \\
& =\left|\left\langle e^{-i \vec{Q} \vec{r}(t=+\infty)}\right\rangle\right|^{2} \\
& =\int G_{s}(\vec{r}, t=+\infty) e^{i \vec{Q} \vec{r}} d \vec{r}
\end{aligned}
$$

$I_{s}(\vec{Q}, t=+\infty)$ est donc le facteur de forme de la portion de l'espace accessible à l'atome (Fig. 4). C'est une grandeur indépendante du temps. On peut donc décomposer $I_{s}(\vec{Q}, t)$ en un terme indépendant du temps, $I_{s}(\vec{Q}, t=+\infty)$, et en un terme fonction du temps. Par transformée de Fourier, on obtient:

$$
S_{\text {inc }}(\vec{Q}, \omega)=I_{s}(\vec{Q}, t=+\infty) . \delta(\omega)+S_{i n c}^{q e}(\vec{Q}, \omega)
$$

où $S_{i n c}^{q e}(\vec{Q}, \omega)$ rend compte de l'intensité quasi-élastique au pied du pic élastique $\delta(\omega)$.

L'intensité du pic élastique d'un spectre de diffusion incohérente de neutrons d'une particule soumise à un mouvement de diffusion est donc étroitement liée au facteur de forme de l'espace de confinement de la particule. On parle de facteur de structure élastique incohérent, ou de façon 

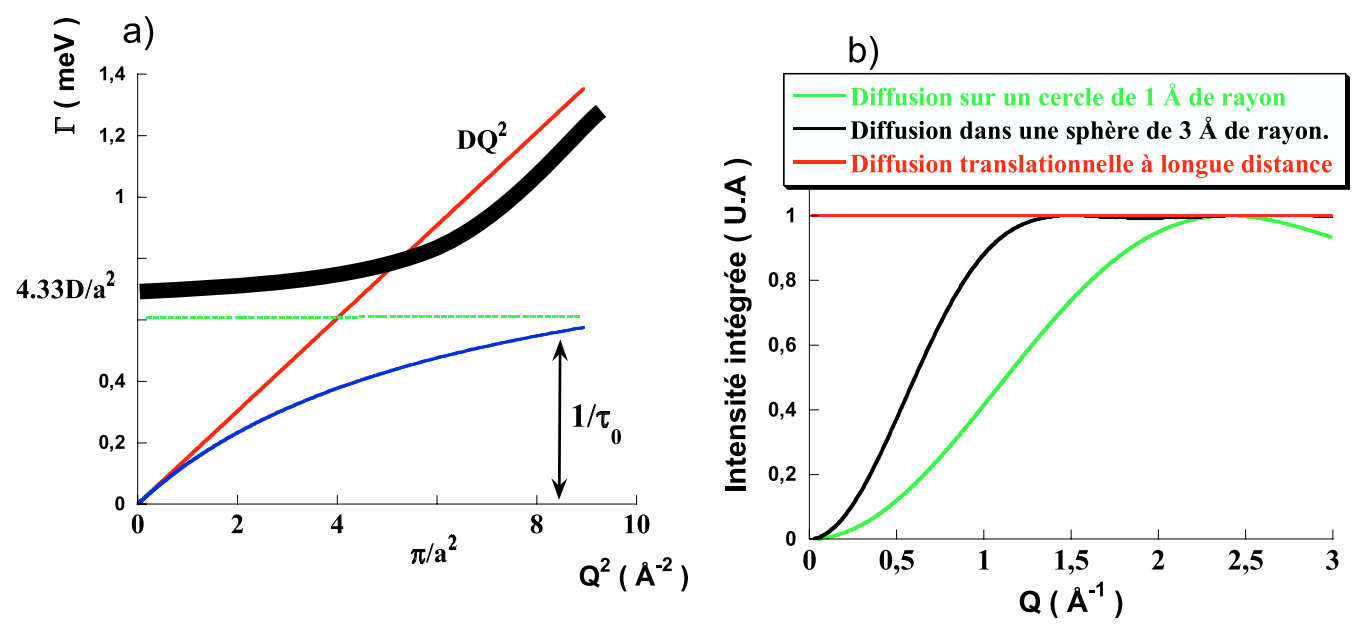

Figure 5. Evolution dans quelques cas choisis de a) la largeur à mi-hauteur et b) de l'intensité intégrée en énergie du signal quasi-élastique (l'intensité élastique a été exclue de l'intégration). Rouge: diffusion à longue distance (loi de Fick ou "loi en $D Q^{2}$ " Eq. 2.26); Bleu: diffusion par sauts (Eq. 2.27); Vert: diffusion réorientationnelle (Eq. 2.33); Noir: diffusion dans un volume de confinement [5, 10].

plus usuelle d'EISF (Elastic Incoherent Structure Factor). Une description plus phénoménologique est donnée Fig. 4.

Détermination expérimentale de L'EISF. Par intégration du facteur de structure dynamique à $\mathrm{Q}$ constant:

$$
\begin{aligned}
\left.\int_{-\infty}^{+\infty} S_{\text {inc }}(\vec{Q}, \omega)\right|_{Q \text { constant }} d \omega & =\int_{-\infty}^{+\infty}\left(\int_{-\infty}^{+\infty} I_{s}(\vec{Q}, t) e^{-i \omega t} d t\right) d \omega \\
& =\int_{-\infty}^{+\infty} I_{s}(\vec{Q}, t) \delta(t) d t \\
& =I_{s}(\vec{Q}, 0)=1
\end{aligned}
$$

$I(Q, \infty)$ est donc selon les équations 2.30 et 2.31 , la fraction élastique de l'intensité diffusée de façon élastique et quasi-élastique. Pour chaque valeur de $\mathrm{Q}$, après décomposition par ajustement numérique du signal total selon un modèle, et extraction des contributions élastique et quasi-élastique, l'EISF est déterminé expérimentalement par le rapport:

$$
\operatorname{EISF}(Q)=\frac{I^{e l}(Q)}{I^{e l}(Q)+I^{q e}(Q)}
$$

où $I^{e l}(Q)$ et $I^{q e}(Q)$ sont respectivement les intensités élastique et quasi-élastique. La comparaison de cette grandeur purement expérimentale à un EISF théorique issu d'un modèle, permet d'accéder à la géométrie des mouvements.

Diffusion rotationnelle. Dans le cas d'une particule diffusant librement sur une sphère de rayon b, le facteur de structure dynamique incohérent s'écrit avec une bonne approximation [6]:

$$
S_{i n c}(Q, \omega)=B_{0}(Q) \delta(\omega)+\left[1-B_{0}(Q)\right] L\left(\omega, \Gamma_{r}\right)
$$

où $D_{R}$ est le coefficient de diffusion rotationnel $B_{0}(Q)=j_{0}(Q b)^{2}$ et $L\left(\omega, \Gamma_{r}\right)$ désigne une lorentzienne en énergie de demi-largeur à mi-hauteur $\Gamma_{r}=2 D_{r}$. Contrairement au cas de la diffusion libre, (i) le fait que la particule soit "confinée" sur une sphère induit l'existence d'un EISF et donc la 
présence d'un pic élastique dans l'expression du facteur de structure dynamique (ii) $\Gamma_{r}$ est indépendant de $\mathrm{Q}$. Il s'agit là d'une propriété commune à tous les mouvements de réorientation.

\subsection{Facteur de structure total}

Le facteur de structure total est le produit de convolution des différents facteurs de structure dynamiques, détaillés, dans les paragraphes précédents:

$$
S_{i n c}(\vec{Q}, \omega)=S_{i n c}^{\text {trans }}(Q, \omega) \otimes S_{i n c}^{r o t}(Q, \omega) \otimes S_{i n c}^{v i b}(\vec{Q}, \omega)
$$

La partie vibrationnelle est composée d'une partie élastique et d'une partie inélastique (Cf. équation 2.19), à laquelle contribuent les vibrations internes de la molécule, $S_{i n c}^{V}(\vec{Q}, \omega)$ et les vibrations externes, dues au réseau $S_{i n c}^{L}(\vec{Q}, \omega)$ :

$$
S_{i n c}^{v i b}(\vec{Q}, \omega)=e^{-2 W(\vec{Q})}\left(\delta(\omega)+S_{i n c}^{V}(\vec{Q}, \omega)+S_{i n c}^{L}(\vec{Q}, \omega)\right)
$$

La partie inélastique $S_{i n c}^{V}(\vec{Q}, \omega)$ est composée d'une série de fonctions $\delta$ centrées sur chacune des fréquences correspondant à un mode de vibration interne de la molécule. La partie $S_{i n c}^{L}(\vec{Q}, \omega)$ prend une forme peu accidentée, assimilable à un bruit de fond plat dans la partie quasi-élastique du spectre.

Dans le cas d'échantillons isotropes, il est possible de faire une moyenne de poudre et le facteur de Debye-Waller prend la forme:

$$
e^{-2 W(\vec{Q})}=e^{-Q^{2}\langle u\rangle^{2} / 3}
$$

$\langle u\rangle^{2}$ est le déplacement carré moyen des atomes, dû aux vibrations internes et aux vibrations externes. Le produit de convolution 2.34 se simplifie:

$$
S_{i n c}(\vec{Q}, \omega)=e^{-Q^{2}\langle u\rangle^{2} / 3}\left(S_{i n c}^{\text {trans }}(\vec{Q}, \omega) \otimes S_{i n c}^{r o t}(\vec{Q}, \omega)+S_{i n c}^{I}(\vec{Q}, \omega)\right)
$$

où $S_{i n c}^{I}(\vec{Q}, \omega)$ est un terme inélastique provenant de la convolution de $S_{i n c}^{V}(\vec{Q}, \omega)$ et $S_{i n c}^{L}(\vec{Q}, \omega)$ par $S_{i n c}^{\text {rot }}(\vec{Q}, \omega) \otimes S_{i n c}^{\text {trans }}(\vec{Q}, \omega)$. Une discussion détaillée des approximations conduisant à ce résultat peut être trouvée à la page 66 de la référence [5]. Dans la partie quasi-élastique du spectre, cette équation se réduit à:

$$
S_{\text {inc }}(\vec{Q}, \omega)=e^{-\vec{Q}^{2}\langle u\rangle^{2} / 3}\left(S_{\text {inc }}^{\text {trans }}(\vec{Q}, \omega) \otimes S_{\text {inc }}^{\text {rot }}(\vec{Q}, \omega)\right)+\mathcal{B}(\vec{Q})
$$

Dans tous ces cas, la réversibilité du temps n'existe pas i.e. $I(Q, t)=I(Q,|t|)$ c'est à dire que les fonctions d'autocorrélations considérées sont paires, et les transformées de Fourier correspondantes sont réelles et paires.

\subsubsection{Conclusions}

Ce chapitre a décrit le facteur de structure dynamique incohérent dans le cas des principaux modes de relaxations dynamiques pertinents dans le cas de macromolécules biologiques. L'intensité diffusée

$$
S(Q, \omega)_{i n c}=S(Q, \omega)_{\text {inc }}^{\text {Quasi }}+S(Q, \omega)_{i n c}^{\text {Ine }}
$$

Le terme $S(Q, \omega)_{i n c}^{\text {Ine }}$ désigne la partie inélastique du spectre à l'origine de laquelle se trouve la dynamique vibrationnelle (intra ou inter moléculaire). $S(Q, \omega)_{\text {inc }}^{\text {Quasi }}$ désigne le terme de diffusion quasi-élastique qui trouve physiquement son origine dans les modes diffusifs ou relaxationnels et admet des profils Lorentzien (i.e. $\left.I(Q, t)_{s}=e^{-t / \tau(Q)}\right)$.

Il s'agit là d'une présentation simplificatrice de la réalité dynamique d'une protéine: il est important de garder à l'esprit que la distinction entre les deux termes peut être difficile dans le cas de modes vibrationnels suramortis. $I(Q, t)_{s}=e^{t / \tau} * \cos (\omega . t) \ldots$ La mesure de l'intensité diffusée en fonction d'une variable d'espace, $Q$, et de temps, $\omega$, permet de sonder simultanément la dynamique (vibrationnelle, 
diffusionnelle) et la géométrie des mouvements. La forme générale du facteur de structure dynamique quasi-élastique incohérent s'écrit:

$$
S(Q, \omega)_{\text {inc }}^{\text {Quasi }}=e^{-Q^{2}\langle u\rangle^{2} / 3}\left\{A_{0}(Q) . \delta(\omega)+\left(1-A_{0}(Q)\right) L(Q, \omega)\right\}
$$

$\langle u\rangle^{2}$ est une mesure de l'extension spatiale des mouvements vibrationnels. L'EISF, $A_{0}(Q)$, permet d'accéder à la géométrie des mouvements (distance de confinement, rayon du cercle sur lequel s'effectue une rotation... ) et l'élargissement quasi-élastique $L(Q, \omega)$ à leur dynamique (coefficient de diffusion). Dans le cas d'une diffusion à longue distance, $A_{0}(Q)=1$ et on n'observe pas de pic élastique (cas d'un liquide).

On peut apporter un complément à l'encadré de la section 2.1.5 pour prendre également en compte la contribution des mouvements diffusionnels dans l'évaluation de l'intensité élastique. De façon très générale:

$$
S(Q, \omega=0)=e^{-Q^{2}\langle u\rangle^{2} / 3} A_{0}(Q) \delta(\omega)+\mathcal{B}(Q)
$$

où $\mathcal{B}(Q)$ représente la contribution du signal quasi-élastique à $\hbar \omega=0$. Nous avons vu que dans le cas d'un système purement vibrationnel harmonique, selon l'équation 2.21, un ajustement linéaire de $\log (S(Q, \omega \approx 0))$ vs $Q^{2}$ permet de déterminer l'extension spatiale, $\langle u\rangle^{2}$, des vibrations. Dans la pratique, il est fréquent d'appliquer cette procédure dans des situations où le système présente un signal quasi-élastique. Il faut cependant être conscient que la valeur de $\langle u\rangle^{2}$ extraite de l'ajustement est alors composite. Au terme $\langle u\rangle^{2}$ purement vibrationnel, vient s'ajouter l'extension spatiale des mouvements diffusionnels qui apparaissent, dans l'équation 2.41, via le terme d'EISF, $A_{0}(Q)$. Sans la prise en compte détaillée du terme $A_{0}(Q)$ qui n'adopte généralement pas une forme gaussienne, les températures de transitions dynamiques des protéines peuvent varier d'une expérience à l'autre en fonction des conditions expérimentales et en particulier de la gamme en $\mathrm{Q}$ utilisée pour les ajustements numériques de $\log (S(Q, \omega \approx 0))$ vs $Q^{2}$.

$S(Q, \omega)_{i n c}$ est la transformée de Fourier sur l'espace et sur le temps de la fonction d'autocorrélation de van Hove qui décrit la dynamique individuelle des particules du système. Dans le cas des systèmes biologiques, $S(Q, \omega)_{i n c}$ donne essentiellement accès à la dynamique individuelle moyenne des protons du système. Le chapitre suivant donne les éléments permettant d'estimer les échelles de temps et d'espace qui, dans la pratique, limitent ces considérations purement théoriques.

\section{INSTRUMENTATION}

Deux types de sources de neutrons existent: les sources à spallation qui délivrent un faisceau pulsé et les sources continues (réacteurs). De façon schématique, dans les deux cas, la distribution en longueur d'onde $\Phi(\lambda)$ vs $\lambda$ des faisceaux délivrés est caractérisée par une contribution maxwellienne de longueur d'onde $\lambda>2 \AA$. Sur sources pulsées, $\Phi(\lambda)$ présente par rapport aux sources continues une contribution additionnelle importante (de l'ordre de $50 \%$ du flux total) de la forme $\Phi(\lambda) \approx 1 / \lambda$ dite contribution éphithermique. Cette forte représentation de neutrons très énergétiques permet (i) d'accéder à la dynamique vibrationnelle intramoléculaire (à haute énérgie $\hbar \omega>1000 \mathrm{~cm}^{-1}$ ) et ce même à très basse température (cf. 2.1.4) et (ii) de mesurer de façon concomitante les événements dynamiques (quasi-élastiques) et structuraux (diffusion aux petits angles et diffraction).

Tous les types de spectromètres inélastiques de neutrons ont été utilisés pour étudier la dynamique des protéines. On recense quelques études de systèmes biologiques mettant à profit les potentialités des spectromètres trois axes. Elles restent cependant à ce jour marginales et nous nous focaliserons ici sur les spectromètres inélastiques utilisant la technique de temps de vol. Dans tous les cas, la partie centrale de l'instrument est constituée de l'échantillon. On distingue alors spectromètre primaire (avant l'échantillon) et secondaire (après l'échantillon). Les fonctions des spectromètres primaires et secondaires varient selon le type de technologie utilisée: géométrie directe ou inversée. Dans les deux 


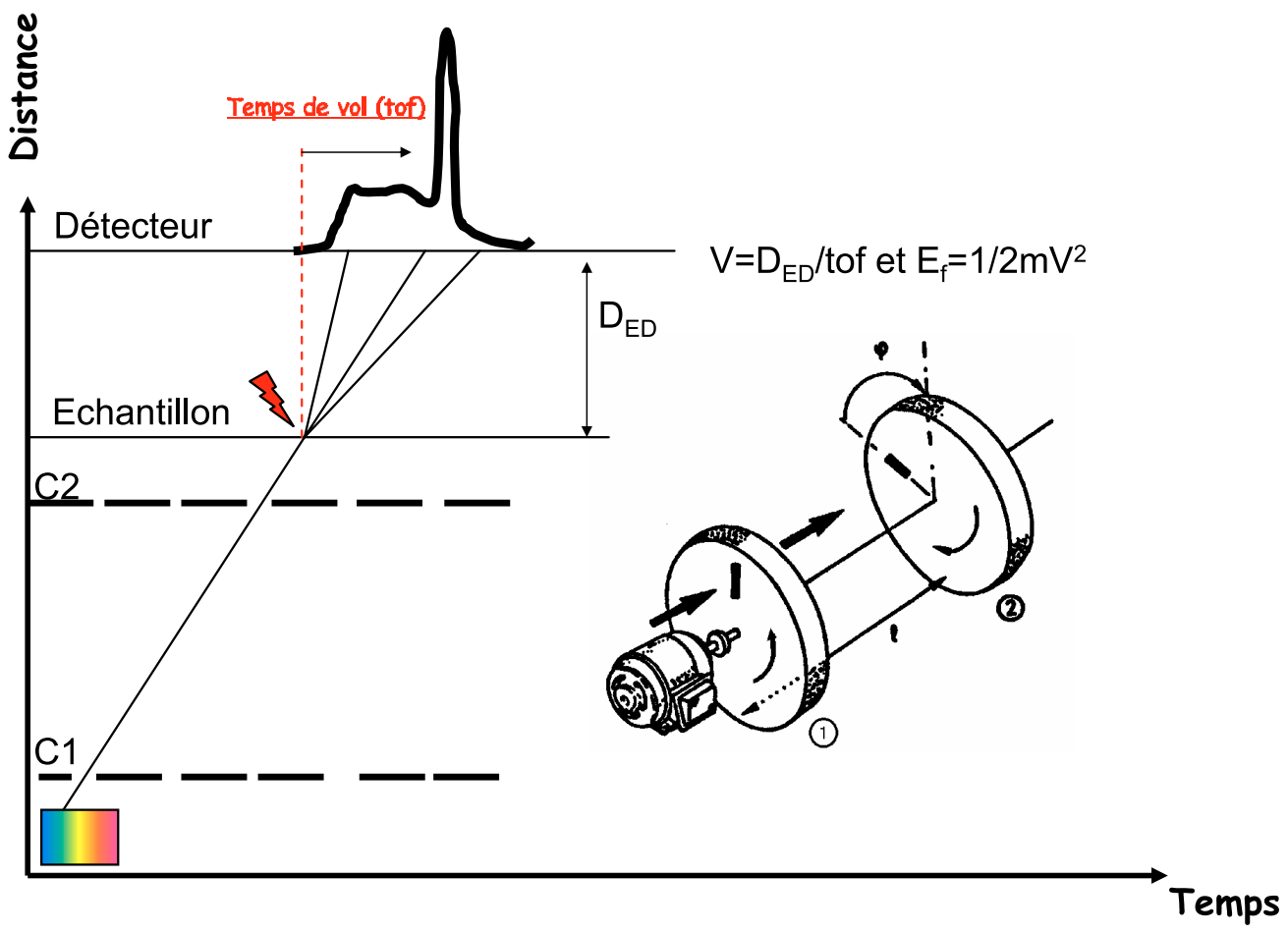

Figure 6. Diagramme des vitesses. Cette figure met en œuvre une technique de représentation dite "diagramme des vitesses". Le temps est porté en abscisse et les distances en ordonnée. Un neutron d'énergie $E$ i.e. de vitesse $v=\sqrt{2 E / m_{n}}$, est représenté par une droite de pente $1 / v$. a) Principe général: cas de la géométrie directe. Pour réaliser une mesure en temps de vol en géométrie directe, il est nécessaire d'extraire, à partir d'un faisceau blanc, des bouffées (pulses) de neutrons monocinétiques. Dans le principe, trois éléments suffisent: deux choppers et un détecteur muni d'une horloge interne. A partir du faisceau blanc continu (carré multicolore) le premier chopper (C1) permet d'obtenir un faisceau blanc pulsé. Le réglage de la phase $\varphi$ du second chopper (C2) permet d'extraire du faisceau blanc délivré par $\mathrm{C} 1$ les seuls neutrons ayant la longueur d'onde et donc l'énergie désirée $E_{0}$. Lorsque ces neutrons atteignent l'échantillon, on déclanche l'horloge du détecteur. Par interaction avec l'échantillon, les neutrons gagnent de l'énergie (augmentation de vitesse, donc de pente sur le graphe), en perdent (diminution de vitesse, donc de pente) ou subissent un choc élastique $(\hbar \omega=0)$ i.e. pas de changement de pente. Ils continuent alors leur trajectoire jusqu'à atteindre le détecteur $\mathrm{D}$ situé à une distance $D_{S D}$ de l'échantillon. A chaque instant on enregistre le nombre de neutrons arrivant sur le détecteur. L'énergie de chaque neutron ayant eu un "temps de vol" $t$ sur la distance SD est $E_{f}=1 / 2 . m_{n}\left(D_{S D} / t\right)^{2}$; ils ont subi un échange d'énergie, $\hbar \omega=E_{0}-E_{f}$. Un calcul élémentaire permet de passer du spectre en temps de vol enregistré sur le détecteur à $S(Q, \omega)$.

cas, l'utilisation de diagrammes des vitesses permet d'appréhender facilement le fonctionnement d'un spectromètre.

\subsection{Temps de vol à géométrie directe}

\subsubsection{Les spectromètres à choppers}

Ce type de spectromètre est plus particulièrement adapté aux sources continues (réacteurs). Dans ce type de technologie, on sélectionne l'énergie des neutrons incidents et on mesure par la technique de temps de vol l'énergie des neutrons diffusés.

Compromis entre flux et résolution. L'une des causes de la résolution en énergie finie, $\Delta E$, des spectromètres par temps de vol est l'existence d'une distribution en longueur d'onde, $\Delta \lambda$, au niveau des 


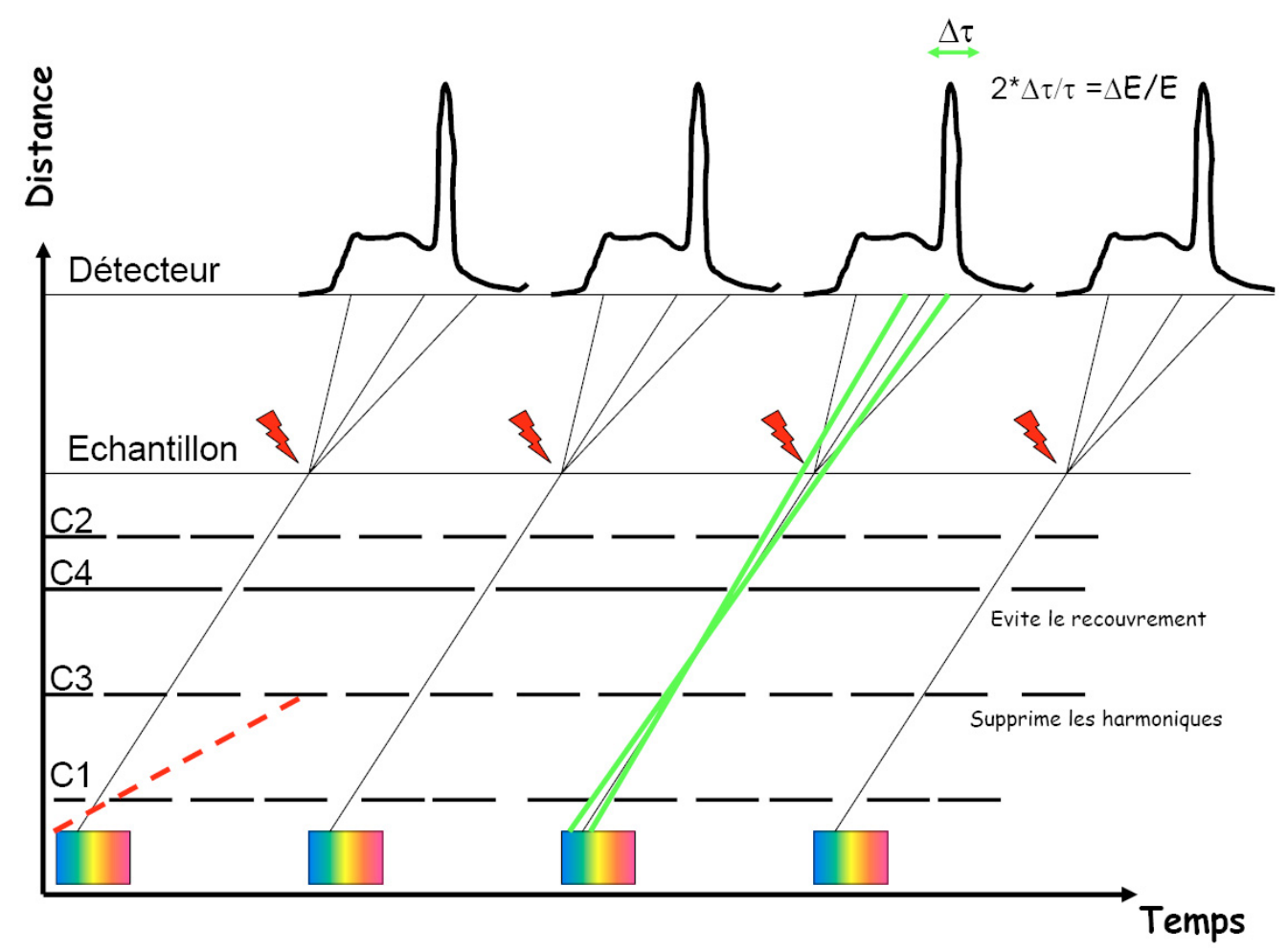

Figure 7. Diagramme des vitesses (suite). Dans la pratique, pour être utilisable, le système nécessite un peu de sophistication.Un troisième chopper $\mathrm{C} 3$ joue le rôle de filtre anti-harmonique: il évite que les neutrons ayant une vitesse sous multiple entière (pointillés rouges) des neutrons $E_{0}$ désirés n'atteignent l'échantillon. Un quatrième et dernier chopper (dit anti-recouvrement ou anti-overlap), C4, ayant une vitesse de rotation sous multiple des autres choppers permet d'espacer dans le temps les bouffées arrivant sur l'échantillon. On s'assure ainsi que les neutrons les plus rapides de la bouffée $\mathrm{n}+1$ diffusés par l'échantillon ne puissent pas rattraper les neutrons les plus lents de la bouffée $n$.

Origine de la résolution finie du spectromètre: Le temps d'ouverture des fenêtres étant fini, il existe une incertitude de l'ordre de quelques pourcents sur l'énergie des neutrons sélectionnés par le système de choppers. Supposons une interaction neutron-échantillon purement élastique $\hbar \omega \approx 0$. L'incertidude sur la vitesse des neutrons incidents produit une incertitude sur leur temps d'arrivée (courbes vertes): le pic élastique n'est pas infiniment fin, mais présente une largeur intrinsèque $\Delta \tau$ : la résolution en énergie de l'instrument. Plus les choppers ont une vitesse élevée, plus le temps d'ouverture des fenêtres devant le guide est bref: l'incertitude sur l'instant de départ diminue, mais le flux également. Quelques exemples de conditions instrumentales sont regroupés Fig. 8.

détecteurs (Fig. 7): $\Delta E / E=2 \Delta \lambda / \lambda=2 \Delta \tau / \tau$ où $\tau$ est le temps de vol des neutrons et $\Delta \tau$, la distribution de temps associée. La solution la plus naturelle pour parvenir à une résolution en énergie de quelques pourcents est de limiter la distribution en énergie des neutrons incidents. C'est la stratégie adoptée dans la conception des spectromètres à "choppers"5. Le prix à payer pour le gain en résolution est le faible flux au niveau de l'échantillon. D'autre part, ces spectromètres ne peuvent occuper qu'une position en bout de guide.

\footnotetext{
${ }^{5}$ Un chopper est un disque absorbant les neutrons dans lequel on a aménagé une ou plusieurs fentes, en rotation dans un faisceaux continu. Les neutrons incidents sont absorbés par le chopper sauf lorsque la fente passe devant le faisceaux. Ce système permet de hacher le faisceaux (to chop en Anglais).
} 

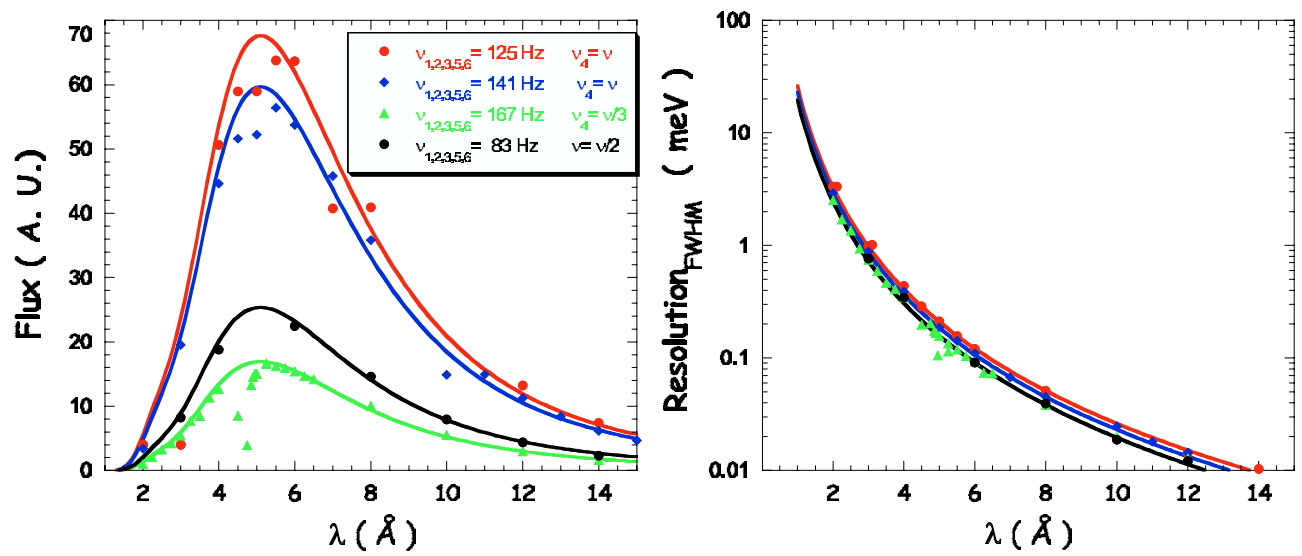

\begin{tabular}{|c|c|c|c|c|c|c|c|c|c|}
\hline & & \multicolumn{4}{|c|}{$v_{4}=v_{1} / 3$ and $v_{2}=v_{1}$} & \multicolumn{4}{|c|}{$v_{4}=v_{1} / 3$ and $v_{2}=v_{1} / 3$} \\
\hline $\begin{array}{c}\mathrm{R} \\
(\mu \mathrm{eV})\end{array}$ & $\begin{array}{c}v_{1,3,5,6} \\
(\mathrm{~Hz})\end{array}$ & $\begin{array}{c}\lambda \\
(\bar{A})\end{array}$ & $\begin{array}{l}Q_{\text {Min }} \\
\left(A^{-1}\right)\end{array}$ & $\begin{array}{l}Q_{\text {Max }} \\
\left(A^{-1}\right)\end{array}$ & $\begin{array}{l}\text { Flux } \\
\text { (A.U.) }\end{array}$ & $\begin{array}{c}\lambda \\
(\hat{A})\end{array}$ & $\begin{array}{l}Q_{\text {Min }} \\
\left(\AA^{-1}\right)\end{array}$ & $\begin{array}{l}Q_{\operatorname{Max}} \\
\left(A^{-1}\right)\end{array}$ & $\begin{array}{l}\text { Flux } \\
\text { (A.U.) }\end{array}$ \\
\hline & 166 & 5.8 & 0.4 & 2.1 & 15.9 & 5.9 & 0.4 & 2.0 & 23.5 \\
\hline 100 & 133 & 6.3 & 0.4 & 1.9 & 18.4 & 6.4 & 0.4 & 1.9 & 26.9 \\
\hline & 83 & 7.3 & 0.3 & 1.6 & 22.8 & 7.5 & 0.3 & 1.6 & 32.7 \\
\hline & 166 & 9.3 & 0.3 & 1.3 & 6.3 & 9.5 & 0.3 & 1.2 & 8.9 \\
\hline 24 & 133 & 10.0 & 0.2 & 1.2 & 6.4 & 10.3 & 0.2 & 1.2 & 9.0 \\
\hline & 83 & 11.8 & 0.2 & 1 & 6.4 & 12.0 & 0.2 & 1.0 & 9.0 \\
\hline
\end{tabular}

Figure 8. Exemples de quelques conditions de résolutions réalisables sur Mibémol. Deux facteurs principaux déterminent la résolution d'un spectromètre à choppers: (i) le temps d'ouverture des fenêtres devant le guide (incertitude sur l'instant de départ des neutrons), ce que contrôle la vitesse de rotation des choppers, et (ii) l'incertitude de la mesure par temps de vol. Ce dernier terme est d'autant plus faible que le temps de parcourt des neutrons est long i.e. que leur longueur d'onde est grande. Ainsi la résolution du spectromètre est régie par deux paramètres de contrôles indépendants: vitesse de rotation des choppers et longueur d'onde incidente. (A gauche): Flux à l'échantillon en fonction de la vitesse des choppers. Pour chacune des courbes, les fréquences des choppers 1,2,3,5,6 sont égales et la fréquence du chopper 4 (anti overlap) est indiquée. (A droite): Résolution en énergie correspondante (FWHM). Les symboles et les couleurs sont les mêmes que pour les deux graphes. Les traits continus correspondent au flux calculé et à la résolution selon [17]. Plus les choppers ont une vitesse élevée, plus le temps d'ouverture de la fenêtre devant le guide est bref: l'incertitude sur l'instant de départ diminue, i.e. la résolution augmente, mais le flux diminue. (En bas) Exemples choisis montrant pour deux résolutions usuelles, $\mathrm{R}=24$ et $100 \mu \mathrm{eV}$ le gain de flux possible, en jouant à la fois sur la longueur d'onde incidente $\lambda$ et la vitesse des choppers. Les calculs ont été effectués en considérant $v_{2}=v_{1}$ et $v_{2}=v_{1} / 3$. Pour éviter tout recouvrement, $v_{4}=v_{1}$ dans les deux cas.

\subsubsection{Temps de vol à géométrie indirecte}

Il existe une alternative à la technologie à choppers: la géométrie "inversée". Le principe consiste à éclairer l'échantillon par un faisceau blanc et à ne détecter par réflexion de Bragg sur un cristal, que les neutrons d'une énergie donnée: dans l'équation 2.3 , on fixe $\hbar \omega_{1}$ et c'est $\hbar \omega_{0}$ qui varie. Cette technologie est particulièrement adaptée aux sources pulsées qui présentent un spectre de longueurs d'ondes incidentes très étendu, de 0.01 à $20 \AA$ Al. Il est possible avec ce type de spectromètres de mesurer des excitations de plusieurs centaines de meV et ce, même à très basse température (cf discussion Chap. 2.1.4).

Un atout: la mesure simultanée de la structure et de la dynamique. Il est possible de disposer dans la base de temps de vol du spectromètre, un détecteur sans intercaler d'analyseur qui sélectionne l'énergie finale $\hbar \omega_{1}$. Les neutrons blancs diffusés par l'échantillon arrivant dans ce détecteur, situé à 

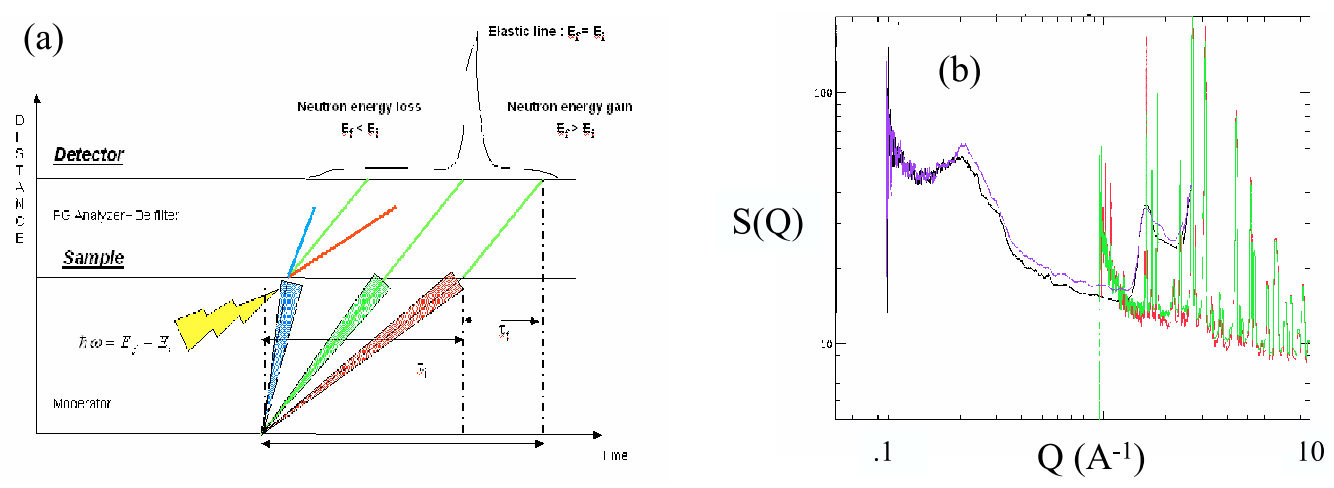

Figure 9. a) Diagramme des vitesses dans le cas d'un spectromètre inélastique à géométrie inversée. b) Spectre de diffraction (QENS, ANL/IPNS) d'une solution de Lysozyme en tampon phosphate $\left(\mathrm{D}_{2} O\right)$ à 100 et 200 $\mathrm{K}$. La première oscillation du facteur de forme du lysozyme (qu'on peut assimiler à un ellipsoïde de révolution) est clairement visible autour de $0.3 \AA^{-1}$. Les pics de Bragg très bien définis à plus grands $\mathrm{Q}$, sont dûs à la cristallisation du tampon phosphate, et non à la protéine qui, étant hydrogénée, diffuse essentiellement de façon incohérente.

un angle de diffusion $2 \theta$, permettent de réaliser une expérience de diffraction i.e. mesurer $^{6} \mathrm{I}(\mathrm{Q})$ vs $\mathrm{Q}$. Certains détecteurs peuvent donc être utilisés en mode diffraction simultanément avec des détecteurs qui conservent le fonctionnement classique d'analyse en énergie. Le spectromètre QENS (Argonne Nat. Lab., Intense Pulsed Neutron Source, Il, USA) permet ainsi de réaliser la mesure du facteur de structure dynamique sur une gamme en $\mathrm{Q}$ de 0.3 à $2.5 \AA^{-1}$ avec une résolution de $85 \mu \mathrm{eV}$ et simultanément de mesurer le facteur de structure statique de $3.10^{-2}$ à $30 \AA^{-1}$. La figure $9 \mathrm{~b}$ donne l'allure d'une telle figure de diffraction dans le cas d'une solution de Lysozyme. Ce type de spectromètre est encore largement sous utilisé en Biologie. Ils permettraient pourtant d'établir une corrélation directe et in situ entre la dynamique d'une protéine et ses changements conformationnels de grandes amplitudes détectés aux petits angles.

\subsection{Conséquence des effets de résolution}

En première approximation ${ }^{7}$, le signal $S(Q, \omega)_{\text {exp }}$ fourni par un spectromètre est le produit de convolution du signal théorique $S(Q, \omega)_{\text {theo }}$ (que l'on mesurerait si l'appareil était infiniment résolutif) par la résolution expérimentale $R(\omega)$ :

$$
S(Q, \omega)_{\exp }=S(Q, \omega)_{t h} \otimes R(\omega)
$$

et donc par transformée de Fourier inverse, en appliquant le théorème de la convolution:

$$
I(Q, t)_{\exp }=I(Q, t)_{\text {theo }} \cdot R(t)
$$

où $R(t)$ est la transformée de Fourier inverse de $R(\omega)$. Généralement, on détermine expérimentalement $R(\omega)$ par la mesure du spectre de diffusion d'un échantillon de vanadium présentant les mêmes caractéristiques géométriques que l'échantillon. Le vanadium étant un diffuseur incohérent, il permet lors de la même mesure de déterminer l'efficacité des détecteurs.

\footnotetext{
${ }_{7}^{6} Q=\frac{4 \pi}{\lambda} \sin (\theta)$ : contrairement au cas "standard", ici $\theta$ est fixe et c'est $\lambda$ qui varie.

7 Lorsque l'on parle de résolution en énergie d'un spectromètre, il s'agit de la résolution "élastique". En réalité la résolution est une fonction du transfert d'énergie. Sur un temps de vol à choppers, la résolution est proportionnelle à l'énergie des neutrons diffusés. L'équation 3.1 n'est qu'une approximation valide dans le domaine faiblement quasi-élastique.
} 

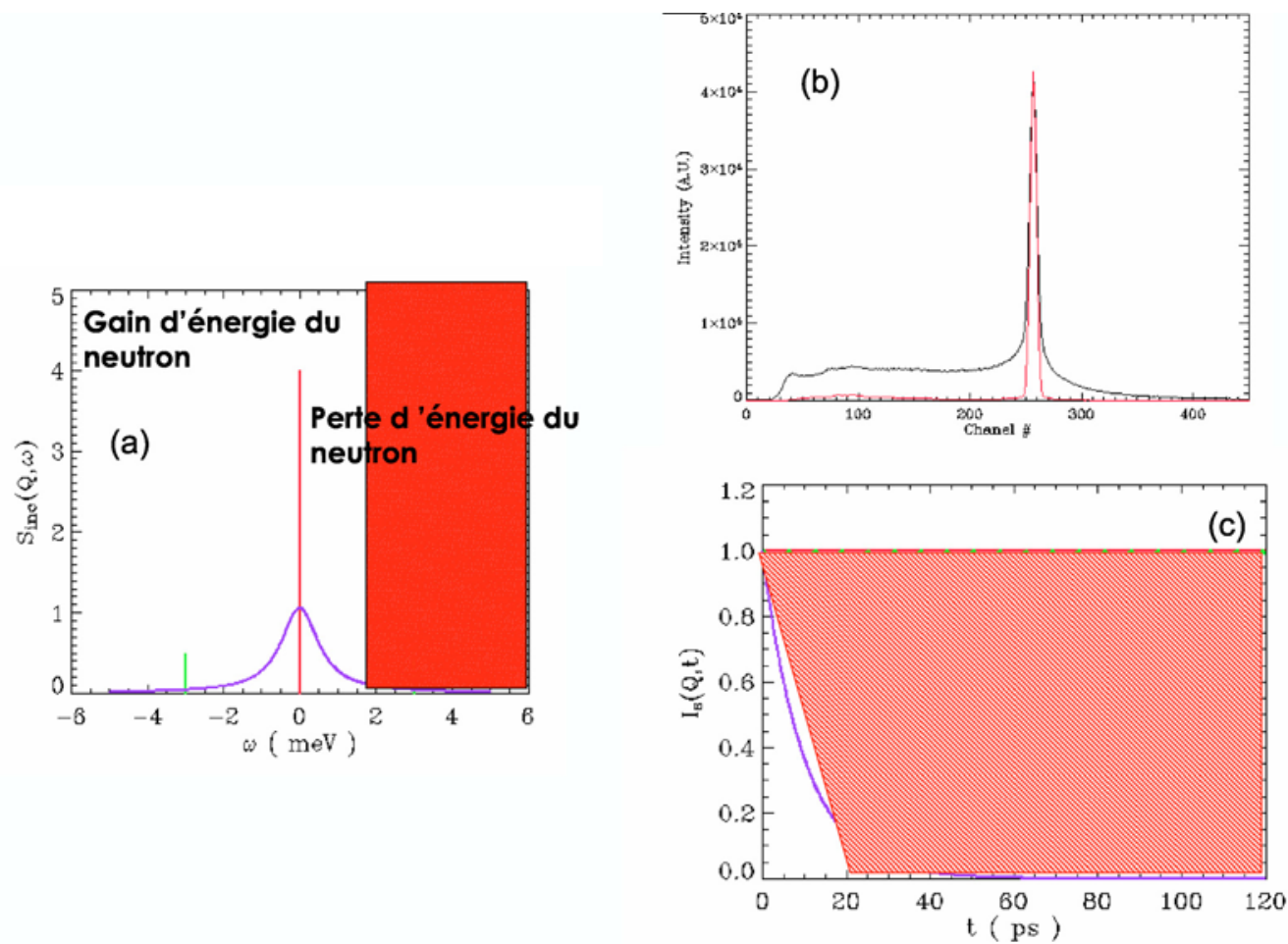

Figure 10. Spectre théorique de diffusion de neutrons et limitations expérimentales. a) L'énergie maximale que peut fournir un neutron à l'échantillon est limitée par sa propre énergie incidente $\hbar \omega_{0}$. La partie rouge du coté perte d'énergie du neutron est donc inaccessible pour des neutrons d'énergie $\hbar \omega_{0}$. b) Spectre expérimental en temps de vol d'une protéine en solution. Les zones élastique, quasi-élastique et inélastique du spectre sont clairement visibles. On constate que la réalité d'une mesure est assez éloignée de la vision idyllique présentée en a) où les raies sont fines et les excitations clairement définies. La complexité du spectre expérimental est dûe en partie à la réalité dynamique de l'échantillon mais aussi à la résolution en énergie intrinsèque du spectromètre (courbe rouge). c) Les considérations instrumentales exposées Fig. 7 ont montré que la résolution en énergie est gaussienne dans l'espace $(\mathrm{Q}, \omega)$. Les transformées de Fourier d'une gausienne et d'une lorentzienne de HWHM $\Delta \omega$ et $\Gamma$ sont respectivement une gausienne en temps de largeur $1 / \Delta \omega$ et une exponentielle décroissante de temps de corrélation $\tau=1 / \Gamma$. Le point à garder en mémoire est que dans les deux cas, la courbe en temps sera d'autant plus large que la courbe en énergie est étroite. Ainsi, la transformée de Fourier du signal quasi-élastique, plus large que la résolution en énergie (courbe b), est un signal temporel plus étroit (exponentielle bleue) que la résolution (courbe rouge). L'équation 3.2 montre que pour mesurer des temps longs, le facteur limitant est le temps maximal correspondant à la résolution. Graphiquement, cela se traduit par le fait qu'il est impossible de mesurer des temps de corrélation correspondant à des exponentielles situées dans le cadran au dessus de la courbe rouge. Cette zone est "interdite" ce que symbolise la zone hachurée en rouge. La seule solution pour mesurer des temps de corrélation plus longs est d'augmenter la résolution en énergie: plus le pic élastique gaussien est étroit en énergie, plus la gaussienne correspondante est large en temps. Cependant, le prix à payer pour une augmentation de la résolution est généralement une baisse importante du flux (Fig. 8).

Pour les processus de relaxation ou les processus gaussiens, l'équivalence temps énergie s'écrit ${ }^{8}$ :

$$
\Delta E(\mathrm{meV})=\frac{0.658}{\Delta t(\mathrm{ps})}
$$

\footnotetext{
${ }^{8}$ Une exponentielle décroissante du temps, présentant un temps de corrélation $10 \mathrm{ps}$, produit donc une lorentzienne en énergie de mi-largeur à mi-hauteur (HWHM) $\Gamma=0.658 / 10=65.8 \mu \mathrm{eV}$
} 
(a)

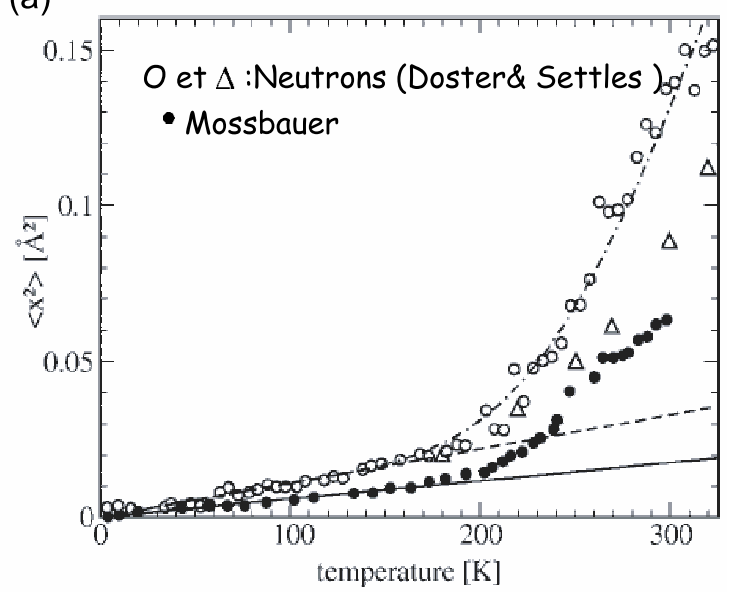

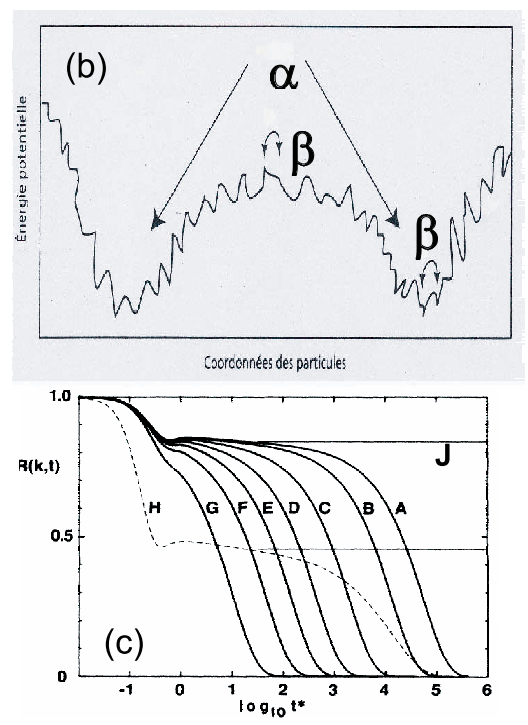

Figure 11. a) Transition dynamique à $180 \mathrm{~K}$, dans la myoglobyne, déterminée par diffusion Mossbauer (•) et diffusion de neutrons ( $\circ$ et $\triangle$ ). Les lignes correspondent à la protéine sèche, i.e. montrent l'absence de transition dynamique en absence de solvant (figure d'après [29]). b) Courbe de potentiel typique d'un verre faible. Les transitions entre minima relatifs proches correspondent aux processus $\beta$, tandis que les transitions de large amplitude et/ou à grande distance correspondent aux relaxations $\alpha$. On sait que ce sont ces dernières qui contrôlent la fonction et on cherche à comprendre comment les fluctuations locales entre minima relatifs proches induisent ces mouvements lents de grandes amplitudes. c) Représentation en fonction de la température de $\mathrm{I}(\mathrm{Q}, \mathrm{t})$ pour un liquide surfondu. La relaxation s'effectue en deux étapes. A temps courts, les processus $\beta$ induisent une faible perte de corrélation. A temps plus long, la localisation des particules dans les cages formées par les proches voisins se traduit, sur la courbe, par la présence d'un plateau. Ces cages ont un temps de vie d'autant plus bref que la viscosité du système est faible et/ou la température élevée. Dans un liquide à haute température, sous l'effet des relaxations $\alpha$, la corrélation retombe vite à 0 (courbe G). Cependant, plus la température diminue, plus le temps de vie des cages s'allonge (courbes $\mathrm{F}$ à $\mathrm{A}$ ). Pour des températures inférieures à une température critique $T_{C}$ (voir texte), le temps de vie de la cage devient infini, les particules restent piegées par leur proches voisins et la courbe $\mathrm{I}(\mathrm{Q}, \mathrm{t})$ est caractérisée par un plateau (courbe $\mathrm{J}$ ) (fig. d'après Bengtzelius, Phys. Rev. A, 34, 5059, 1986).

Ces effets de résolution impliquent qu'en diffusion de neutrons, l'observation et les grandeurs qui y sont associées (distances, temps de corrélation) ne sont valables qu'à la résolution du spectromètre près. Un exemple de ce principe est donné plus loin dans le cas de l'étude expérimentale de la parvalbumine (cf 5).

\section{UNE PROTÉINE EST-ELLE UN VERRE ?}

\subsection{Transition dynamique et fonction}

Cette analogie à priori incongrue entre un verre et un objet biologique, a été suscitée par une expérience fondatrice pour l'étude de la dynamique des protéines. En 1982, en mesurant sur une échelle de temps de quelques dizaines de ns, par spectroscopie Mössbauer, la dépendance en température du déplacement carré moyen du fer de l'hème de cristaux de deoxymyoglobine, Parak et al. [13] ont mis en évidence une transition dynamique à $180 \mathrm{~K}$. Cette transition a également été observée par diffusion inélastique de neutrons [14] dans la gamme de temps des picosecondes sur une poudre de protéine hydratée. Dans les deux cas, la "transition dynamique" est caractérisée par un écart à la linéarité du déplacement carré moyen à la température de transition (Fig. 11). Comme attendu dans le cadre de la section 2.1.5 on observe en parallèle une augmentation significative de l'intensité diffusée à faible transfert d'énergie 
(densité d'états vibrationnels) et dans la zone quasi-élastique du spectre. Il ne s'agit cependant pas d'une transition de phase puisque la structure de la protéine, observée en parallèle par diffusion des rayons X, n'est pas modifiée à la transition. Enfin, et c'est le point clé de cette observation, la transition dynamique est étroitement corrélée avec l'apparition de l'activité de la protéine mais n'est pas observée sur la protéine sèche démontrant le lien étroit entre phénomènes d'hydratation, dynamique et fonction des macromolécules biologiques. Ces observations ont depuis été faites sur d'autres protéines solubles et membranaires $[18,19]$ confortant le caractère général de la transition dynamique dans le domaine de la biophysique. Le point essentiel est que cette expérience a permis de déterminer non seulement une corrélation très nette entre la dynamique d'une protéine et sa fonction, mais aussi de montrer le rôle central de l'hydratation.

Ce comportement d'écart à la linéarité à la température de la tranisiton dynamique est un phénomène également observé dans le cas des systèmes vitreux au dessus de la température de transition vitreuse $T_{g}$. Cette similarité de comportement conduit naturellement à la question: "une protéine est-elle un verre?"

Un argument fort qui soutient cette analogie est la compacité des protéines: dans un système aussi dense, les changements conformationnels ne peuvent avoir lieu que de façon concertée comme c'est le cas dans un verre ou un liquide surfondu. Nous rappellons quelques propriétés de ces sytèmes.

\subsection{Propriétés des verres et des liquides surfondus}

Les verres et liquides surfondus présentent plusieurs caractéristiques communes [24]:

- Comportement non-Arrhénius des cinétiques de relaxation. Afin de permettre d'établir une classification cartésienne des liquides surfondus, Angell a proposé un mode de représentation unificateur de leur viscosité: les observables (viscosité ou temps de relaxation) sont représentées non pas en fonction de $1 / \mathrm{T}$ mais de $T_{g} / T$. L'expérience montre que les systèmes réels s'inscrivent entre deux cas limites:

- liquides forts: les observables suivent un comportement Arrhénius. La silice en est l'exemple classique. La structure locale est régie par des liaisons covalentes fortes. Les déplacements d'atomes ne sont possibles que par sauts thermiquement activés.

- liquides fragiles: certains systèmes présentent un comportement fortement non-Arrhénius. Les données peuvent se mettre sous la forme $\eta=\eta_{0} \exp \left[D \cdot T_{0} /\left(T-T_{0}\right)\right]$ où le paramètre $T_{0}$ permet de décrire la forte augmentation d'énergie d'activation à $T_{g}$. D est la mesure de l'énergie d'activation du système à $T_{g}$. Plus $\mathrm{D}$ est élevé, plus le liquide est fort. On utilise également $m \propto 1 / D$ comme indice de fragilité du système. L'orthoterphényle est l'exemple canonique de liquide fragile: la structure de ce liquide moléculaire est essentiellement régie par des interactions faibles de type Van der Waals.

- le caractère non exponentiel des relaxations. Une seconde caractéristique des liquides surfondus et des verres est le caractère non exponentiel des relaxations:

$$
\tau=\tau_{0} e^{-\left(\frac{t}{\tau}\right)^{\beta}}, \quad 0<\beta<=1
$$

Le cas $\beta=1$ correspond à un temps de relaxation $\tau$ unique. Les systèmes présentant une distribution de temps de relaxation peuvent être décrits par des valeurs de $\beta$ inférieures à 1 . Plus $\beta$ est faible, plus la distribution de temps de relaxation est large.

- Nonlinéarité des relaxations. Dans le régime non ergodique, la relaxation dépend de l'écart au point d'équilibre. On parle de vieillissement (“ageing” en anglais).

Un point particulièrement intéressant de la physique des verres est qu'elle peut s'appuyer sur des outils théoriques. La théorie de couplage de mode (Mode Coupling Theory ou MCT) [28] permet en particulier de décrire la relaxation en deux étapes expérimentalement observées. La relaxation dite " $\beta$ ", 

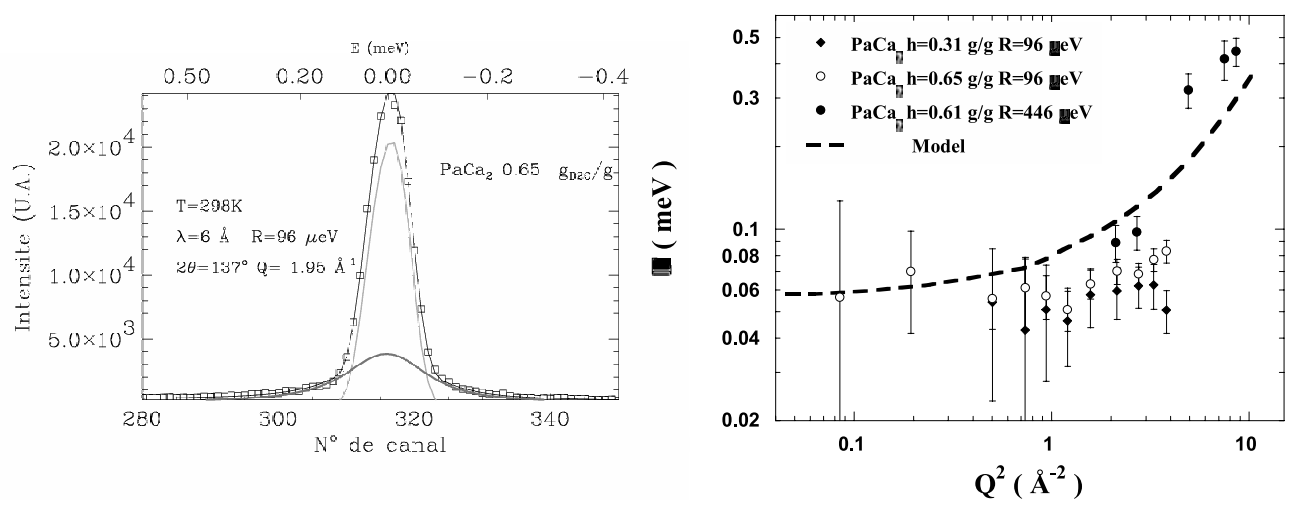

Figure 12. a) Spectre de diffusion inélastique de neutrons (Mibémol, LLB) de poudre de parvalbumine hydratée à $0.65 \mathrm{~g} / \mathrm{g}(\mathrm{R}=96 \mu \mathrm{eV})$ ajusté numériquement par un pic élastique et une lorentzienne quasi-élastique de HWHM $\Gamma$. b) Evolution de $\Gamma$ en fonction de Q pour deux taux d'hydratation $0.31 \mathrm{~g} / \mathrm{g}$ et $0.65 \mathrm{~g} / \mathrm{g}$. $\Gamma$ présente un plateau à petits $\mathrm{Q}$, suivi d'une zone de dépendance en $\mathrm{Q}$. Ce comportement est caractéristique d'un mouvement de diffusion dans un espace de confinement de rayon a (cf. Fig. 5a). Le pointillé est le modèle calculé pour coefficient de diffusion $\mathrm{D}=7.10^{-6} \mathrm{~cm}^{2} / \mathrm{s}$ et $\mathrm{a}=1.7 \AA$

qui intervient aux temps courts, correspond à une relaxation rapide des particules dans la cage formée par les proches voisins.

La densité locale élevée induit un phénomène de localisation qui se traduit en terme de fonction de corrélation par la présence d'un plateau. A haute température, sous l'effet de phénomènes collectifs cette cage finit par relaxer et la corrélation redevient nulle. Cet effet est la traduction en terme de fonction de corrélation des relaxations $\alpha$ évoquées plus haut. Le temps de corrélation associé, $\tau_{\alpha}$ est relié à la viscosité du système. En dessous d'une température critique $T_{C} \approx 1.2 T_{g}$, l'énergie du système n'est pas assez élevée pour permettre à la cage de relaxer et la localisation devient définitive: on parle de verre et de perte d'ergodicité. La MCT permet de relier $\tau_{\alpha}, T_{C}$ et $\beta$.

$$
\tau_{\alpha}(T) \propto\left(T-T_{C}\right)^{\gamma(\beta)}
$$

\subsection{Un exemple d'approche statistique de la dynamique des protéines : la dynamique du lysozyme}

Sokolov et collaborateurs, étudient la dynamique des protéines par diffusion de neutrons et diffusion de la lumière. Par une mesure de la dynamique sur une large bande de fréquence, ils montrent que le processus de relaxation de la protéine s'effectue en deux étapes. Dans un premier temps, le système relaxe par un processus rapide (dans la gamme de temps des picosecondes) peu affecté par la température, puis par un processus lent qui est, lui, fortement tributaire de la température. Il s'agit exactement du scenario prédit par la théorie de couplage de modes. Il s'agit d'une approche statistique qui ne prétend pas identifier un mouvement précis et donc relativement éloignée mais très complémentaire de l'analyse en modes normaux évoquée plus haut (2.1.5).

En étudiant l'évolution de la transition dynamique en fonction de co-solvants, ces auteurs montrent qu'il existe une corrélation étroite entre la dynamique de la protéine et celle du solvant, suggérant que c'est le solvant qui impose la dynamique de la protéine.

Cela suggère le rôle primordial assuré par les premières couches d'hydratation, c'est à dire l'eau interfaciale. Ainsi, une transition liquide-liquide a été mise en évidence dans le cas de l'eau en monocouche à la surface d'un milieu hydrophile [27]. En mettant à profit la mesure simultanée de l'intensité diffusée en $Q$ et $\omega$, il a de plus été possible de scinder la dynamique de l'eau interfaciale en contributions rotationelle et translationnelle. On montre que la transition dynamique du lysozyme 
hydraté en monocouche est d'abord induite par une transition mettant en jeu la dynamique rotationelle des molécules d'eau interfaciale.

Il est possible de décrire la dynamique des protéines dans le cadre de la théorie de couplage de modes, ce qui est habituellement l'une des signatures d'un liquide fragile. Dans le même temps, comme un verre fort, les protéines présentent un pic Boson (excès d'intensité - autour de $3 \mathrm{meV}$ - correspondant à des modes localisés et ce, même au dessus de la température de transition dynamique). Enfin, on n'observe aucun saut de chaleur spécifique lors du passage d'une protéine par la température de transition dynamique. Si l'on s'en tient à la classification habituelle des verres, les protéines sont des sytèmes vitreux à la fois "forts" et "fragiles".

\section{LA DYNAMIQUE DE LA PARVALBUMINE. UNE APPROCHE MÉCANISTIQUE}

La parvalbumine $(\mathrm{Pa})$ est une petite protéine de $11 \mathrm{kDa}$ dont la structure est caractérisée par la présence d'un coeur hydrophobe et de deux sites de fixation d'ions divalents. La parvalbumine exerce ainsi un rôle important dans la régulation des concentrations intracellulaires de $\mathrm{Mg}^{2^{+}}$et de $\mathrm{Ca}^{2+}$ pendant le cycle contraction-relaxation musculaire.

L'influence de l'hydratation et de la nature des ions divalents sur la dynamique de la parvalbumine ont été étudiés conjointement par diffusion inélastique incohérente de neutrons et RMN du solide $\left({ }^{13} \mathrm{C}\right.$ en abondance naturelle et rotation à l'angle magique).

Les échantillons étaient des poudres de parvalbumine échangées (dialyse contre $\mathrm{D}_{2} \mathrm{O}$ pour échanger les protons labiles) puis hydratées ${ }^{9}$ par $\mathrm{D}_{2} \mathrm{O}$ en phase vapeur à divers taux d'hydratation de textrmh $=0$ (protéine sèche) à $\mathrm{h}=0.7 \mathrm{~g} / \mathrm{g}$. Certaines molécules d'eau font partie intégrante de la structure de la protéine si bien que la masse de protéine sèche n'est définie qu'à quelques pourcents près.

Lors de ces expériences, la parvalbumine étant hydratée par $\mathrm{D}_{2} \mathrm{O}$, on observe par diffusion inélastique de neutrons la dynamique de la protéine échantillonée par la dynamique individuelle moyenne de ses protons. Les experiences ont été menées sur le spectromètre en temps de vol Mibémol du LLB. Une première expérience a été menée avec une résolution de $96 \mu \mathrm{eV}$, sur une gamme en $\mathrm{Q}$ de 0.3 à $2 \AA^{-1}$. L'ajustement des spectres avec un pic élastique et une lorentzienne de largeur à mi-hauteur $\Gamma$ est satisfaisant. On observe que l'intensité quasi-élastique est fortement dépendante de l'hydratation de la protéine. Les EISF mesurés pour chacune des hydratations sont donnés Fig. 12 . De $\mathrm{h}=0$ à $\mathrm{h}=0.4, \Gamma$ est indépendant de Q. A $\mathrm{h}=0.65$, une zone de dépendance en Q semble apparaître, cependant l'incertitude des mesures ne permet pas d'être affirmatif. Les spectromètres à choppers permettent d'accéder à une large gamme de conditions expérimentales (énergie, gamme en Q). Cette possibilité offerte par Mibémol a été mise à profit pour rélaiser une expérience utilisant une longueur d'onde incidente de $4 \AA$ afin d'accéder à des valeurs de $\mathrm{Q}$ plus élevées. Cette expérience a permis de confirmer que $\Gamma$ présentait effectivement une zone de dépendance en Q.

Ce comportement est une caractéristique d'un phénomène de diffusion dans un espace confiné. L'analyse de l'EISF $\left(E I S F=I_{E l} /\left(I_{E l}+I_{Q u a s i}\right)\right)$ permet d'acceder à la forme de cet espace de confinement. Les protéines sont caractérisées par une grande richesse dynamique. On ne peut expérimentalement accéder qu' aux temps de corrélation de $15 \mathrm{ps}$ au plus. Les temps plus longs donneront des contribution élastiques (cf. discussion10). Soit p, la fraction de protons de la protéine ayant des temps de corrélation plus longs que $1 / \mathrm{R}$, les protons de la fraction complémentaire (1-p) apparaîssent mobiles. L'EISF total s'écrit donc:

$$
E I S F=p+(1-p) A_{0}(Q)
$$

\footnotetext{
${ }^{9}$ L'hydratation est définie comme étant la masse d'eau par masse de protéine sèche.
} 

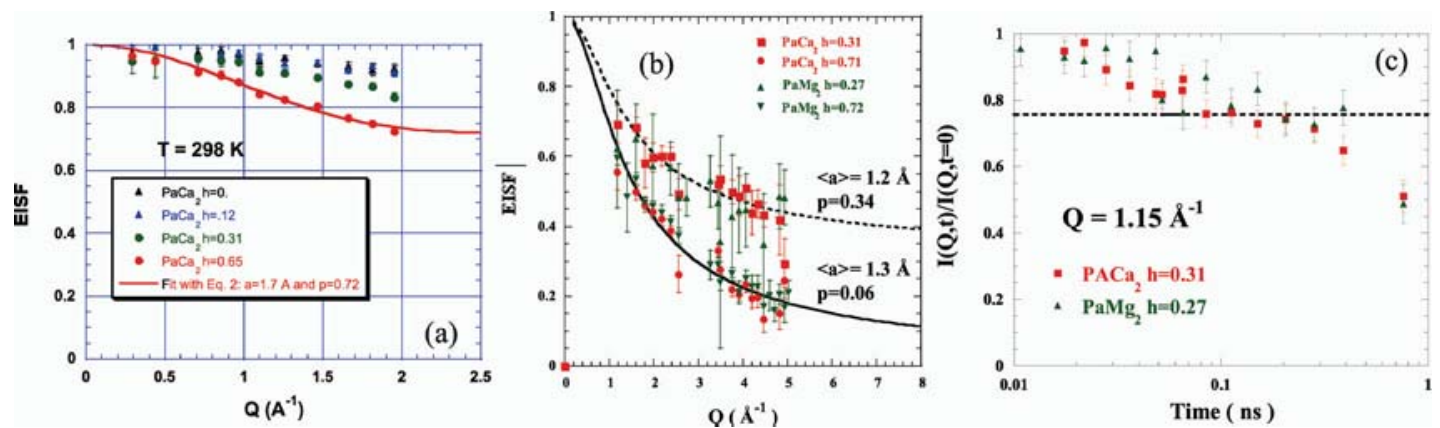

Figure 13. Effets de résolution sur la dynamique de la parvalbumine [23]. Pour une échelle de temps donnée (résolution en énergie du spectromètre), on décrit la dynamique du système comme la somme de deux contributions: une fraction $\mathrm{p}$ des protons de la protéine sont considérés comme immobiles (temps de corrélation significativement plus longs que l'échelle d'observation) tandis que les 1-p protons perçus comme mobiles diffusent librement dans une sphère de confinement de rayon a. a) Sur une échelle de temps de 15 ps accessible sur Mibémol à $6 \AA$ A $(\mathrm{R}=100$ $\mu \mathrm{eV}$ ), à $\mathrm{T}=298 \mathrm{~K}$, pour une hydratation de $0.65 \mathrm{~g} / \mathrm{g}$, seuls $1-0.72=28 \%$ des protons sont perçus comme mobiles et diffusant dans une sphère de confinement de rayon $1.7 \AA$. b) EISF de la parvalbumine en fonction du taux d'hydration et de l'ion divalent. Des mesures ont été effectuées sur IN13 $(\mathrm{R}=15 \mu \mathrm{eV})$ à $\mathrm{T}=278 \mathrm{~K}$. Les lignes pleines et pointillés représentent l'ajustement selon l'équation 5.1, à hydratation respectivement faible $(0.27 \mathrm{~g} / \mathrm{g})$ et forte $(0.72 \mathrm{~g} / \mathrm{g})$. Les valeurs de $\mathrm{p}$ et a sont indiquées dans chaque cas. Sous l'effet de l'hydratation, $\mathrm{p}$ diminue fortement de 0.34 à 0.06 . De plus, par comparaison avec la courbe a), on constate qu'à hydratation comparable $(0.65$ et $0.72 \mathrm{~g} / \mathrm{g}$ ), si l'on observe le système à meilleure résolution i.e. à temps plus long, la fraction de protons perçus comme immobiles diminue ( 72 contre $6 \%$ ) et le rayon de confinement diminue (1.7 contre $1.3 \AA$ ). Ceci traduit le fait que sur une échelle de temps de $150 \mathrm{ps,} \mathrm{pour} \mathrm{une} \mathrm{hydratation} \mathrm{de} \mathrm{la} \mathrm{protéine} \mathrm{correspondant} \mathrm{à} 3$ monocouches, la presque totalité des atomes apparaîssent mobiles. Cependant, les atomes du coeur hydrophobe de la protéine, qui contribuent maintenant au signal, subissent un encombrement stérique plus important que les atomes des chaînes latérales de surface, si bien que le rayon de diffusion moyen diminue. c) Il est possible de mesurer la dynamique de la protéine dans la gamme de temps des ns par echo de spin de neutrons (IN11-ILL). Par définition (Eq. 2.30 et Fig. 4.c) un EISF apparaît, sur une courbe $I(Q, t)$, comme un plateau indépendant du temps. La valeur de l'EISF mesuré pour $\mathrm{h}=0.31$ sur IN13 à $\mathrm{Q}=1.15 \AA^{-1}$ (fig. b), est reportée en pointillés. Comme attendu, sur une échelle de temps commune de $150 \mathrm{ps}$, les deux mesures (valeur de $\mathrm{I}(\mathrm{Q}, \mathrm{t})$ pour un temps t donné) concordent. À temps plus long, la diminution du I(Q,t) expérimental sous la valeur de l'EISF mesuré sur IN13, suggère que des modes relaxationels, non perçus à une échelle de $150 \mathrm{ps}$ apparaissent dans la gamme de temps des nanosecondes.

Afin de décrire le plus simplement possible l'espace de confinement, nous l'assimilerons à une sphère de rayon a. $A_{0}(Q)$ prend la forme [10]:

$$
A_{0}(Q)=\left[\frac{3 j_{1}(Q a)}{Q a}\right]^{2}
$$

où $j_{1}$ est la fonction de Bessel sphérique de première espèce d'ordre 1: $j_{1}(x)=\frac{x \cos (x)-\sin (x)}{x^{2}}$. Un ajustement numérique de l'EISF de $P a C a_{2} \mathrm{~h}=0.65$ conduit à a $=1.7 \AA$ et $\mathrm{p}=0.72$ : sur une échelle de temps de $15 \mathrm{ps}, 28 \%$ des protons de la protéine diffusent dans un espace de confinement assimilable à une sphère de $1.7 \AA$ de rayon. La question qui se pose alors est d'identifier ces protons mobiles puis de donner une interprétation physique à leur sphère de confinement.

Considérons un atome d'hydrogène d'une chaine latérale de lysine. La chaîne latérale qui le porte est dans une conformation particulière, subissant l'influence de la réorganisation incessante du réseau de liaison hydrogène de l'eau d'hydratation, la chaîne aliphatique peut adopter une autre conformation rotamère particulière, et ce jusqu'à la prochaine réorganisation locale du réseau. À chaque réorientation de la chaîne, un atome d'hydrogène réalise un mouvement librationnel ou un mouvement de diffusion par sauts très rapides autour de la liaison $\mathrm{C}-\mathrm{C}$ qui le porte. Le nombre de sites est difficile à déterminer: un saut sur deux sites semble le plus probable. La combinaison d'une telle réorientation autour de l'axe 
$\mathrm{C}-\mathrm{C}$ qui le porte, avec la réorientation de cette liaison, autour d'un axe constitué par une liaison C-C en aval ou en amont, conduit l'atome d'hydrogène à adopter un mouvement à la géométrie très complexe.

Le mouvement que nous observons sur une échelle de temps de quelques ps, n'est donc qu'une vision moyenne des mouvements de réorientation multiples. Cette trajectoire peut être décrite par la diffusion dans une sphère. Cela justifie le rayon relativement faible de la sphère: la rotation autour des axes C-C se fait sur un rayon de $1 \AA$. Si le mouvement en question est un mouvement de libration suramorti, on peut penser que la distance associée est encore plus faible.

Carpentier et al. [20] observent le même phénomène pour les protons d'une chaîne alkyle dans le tétrapalmitate cuivreux, deutérié sélectivement. Les atomes d'hydrogène liés à un carbone près du cœur de cette molécule ont des mouvements de faible amplitude. Les autres atomes d'hydrogène des chaînes latérales, adoptent un comportement de diffusion dans une sphère dont le rayon augmente linéairement entre le premier et le cinquième carbone, où il atteint la valeur limite de $3 \AA$. Une distance de $1.7 \AA$ est trouvée dans leur système, pour les atomes d'hydrogène fixés à la troisième liaison $\mathrm{C}-\mathrm{C}$, ce qui est cohérent avec la longueur des chaînes latérales de lysine.

En conclusion, la sphère de confinement n'est qu'un moyen simple de décrire l'enveloppe de la trajectoire des protons des chaîne latérales de surface de la protéine.

Cette étude montre les possibilités de la méthode, elle en montre aussi les limites:

- L'information dynamique est "moyennée”. Il est donc particulièrement intéressant de coupler la diffusion de neutrons qui donne une information globale et la RMN qui permet de sonder la dynamique site à site.

- Sensibilité: à ce jour, il a été impossible de détecter, une différence notable dans la dynamique des formes calciques et magnésiques de la parvalbumine.

\section{CONCLUSION}

La diffusion inélastique de neutrons permet, notamment, de mettre à profit des effets isotopiques, pour accéder à la dynamique de la protéine en masquant celle du solvant, ou inversement. L'information dynamique est mesurée en terme de fonctions d'autocorrélation des positions des atomes d'hydrogène de la protéine (ou du solvant). L'échelle de temps mis en jeu s'étend de quelques fractions de picosecondes à quelques nanosecondes. Il est possible de décrire la dynamique du système à l'aide d'un modèle très simple qui décrit la dynamique complexe d'une protéine en terme de diffusion d'une fraction des protons dans un espace de confinement. Ce modèle a été étendu au traitement de la dynamique des protéines en solutions [26].

On peut considérer que la description de la dynamique des protéines est un domaine de la science encore balbutiant. Sans être la solution miracle, il semble clair que les stratégies de deutération spécifique ouvriront la voie à des avancées majeures. Les approches par manipulation de molécules uniques semblent également très prometteuses. En parallèle, des dévelop-pements théoriques existent en termes de modes localisés non dispersifs (breathers) [21, 22]. Ces approches complémentaires sont souhaitables pour, à terme, pouvoir disposer d'une "théorie de la dynamique des protéines", et ainsi espérer le même type de développement qu'a connu la physique des polymères avec l'introduction des lois d'échelle.

\section{Références}

[1] G. L. Squires, Introduction to the theory of thermal neutron scattering, Cambridge University Press (1978).

[2] L. van Hove, Correlations in space and timeand Born a pproximation scattering in systems of interacting particles, Phys. Rev. 95, 1954, 249-262.

[3] V. F. Sears, Neutron scattering lengths and cross sections, Neutron News 3, 1992 26-37.

[4] M. Bée, Physica B 182, 1992, 323-336. 
[5] M. Bée, Quasielastic neutron scattering: principles and applications in solid state chemistry, biology and materials science, Adam Hilger, Bristol and Philadelphia (1988).

[6] V. F. Sears Theory of cold neutron scattering by homonuclear diatomic liquids, Can. J. Phys. 44, 1966, 1279.

[7] S. Yip, Quasi-elastic scattering in neutron and laser spectroscopy, in Spectroscopy in biology and chemistry: neutrons, Xray, laser, S. H. Chen and S. Yip Eds., Academic Press (1974).

[8] J.F. Gibrat, N. Go, Proteins. 1990;8(3):258-79.

[9] J.-M. Zanotti, M.-C. Bellissent-Funel and J. Parello, Biophys. J., 76, 2930 (1999).

[10] F. Volino and A. J. Dianoux, Mol. Phys., 41, 271, (1980).

[11] J.P. Declercq, B. Tinant, J. Parello and J. Rambaud, J. Mol. Biol., 220, 1017 (1981).

[12] L. Carpentier, M. Bée, A.M. Giroud-Godquin, P. Maldivi and J.C. Marchon, Mol. Phys., 68, 1367 (1989).

[13] F. Parak, E.W. Knapp, and D. Kucheida. 1982. J. Mol. Biol. 161:177-194.

[14] W. Doster, S. Cusack, and W. Petry. 1989. Nature. 337:754-756.

[15] X. Zhuang, Science, 296, 2002.

[16] A. Ansari, J. Berendzen, S. F. Bowne, H. Frauenfelder et al. Proc. Natl. Acad. Sci. USA. 82:5000 (1985).

[17] R. Lechner, Physisca B, 180-181, 973(1992).

[18] M. Ferrand, A. J. Dianoux, W. Petry and G. Zaccaï, Proc. Natl. Acad. Sci. USA, 90, 28, 9668-9672 (1995).

[19] C. Andreani, A. Filabozzi, F. Menzinger, A. Desideri, A. Deriu, D. Di Cola, Biophys. J., 68, 2519-23 (1995).

[20] L. Carpentier, M. Bée, A. M. Giroud-Godquin, P. Maldivi and J.C. Marchon, Mol. Phys., 68 (6), 1367-78 (1989).

[21] A. Xie, L. van der Meer, W. Hoff, R.H. Austin, PRL, 84, 5435 (2000).

[22] S. Aubry, G. Kopidakis, Journal of Biological Physics, soumis (2005).

[23] J.-M. Zanotti, Bellissent-Funel et J. Parello, Applied Physics A, 74:S1277-S1279 (2002).

[24] J. L. Green, J. Fan and C. Angell, Journal of physical chemistry, 98 (1994).

[25] G. Caliskan, A. Kisliuk and A.P. Sokolov, Journal of non crystalline solids, 307-310, 868-873 (2002).

[26] J. Pérez, J.-M. Zanotti and D. Durand, Biophys. J., 77, 454-469 (1999).

[27] J.-M. Zanotti and Bellissent-Funel, Europhysics letters, 71, 1:7 (2005).

[28] W. Gotze, L. Sjogren, Rep. Prog. Phys., 55, 241 (1992).

[29] F. Parak et al., Rep. Prog. Phys. 66, 103129 (2003). 